\title{
Security-Oriented Trellis Code Design for Spatial Modulation
}

\author{
Mingxi Yin, Yuli Yang, Senior Member, IEEE, and Bingli Jiao, Senior Member, IEEE
}

\begin{abstract}
To achieve physical layer security (PLS) with a flexible implementation, a novel design named security-oriented trellis coded spatial-modulation (SO-TCSM) is proposed in this paper, where the transmitter does not know the channel state information (CSI) of wiretapping channels and relies on the legitimate CSI to vary the mapping patterns for the antenna information and the radiated information, aiming at the optimisation of free Euclidean distances between the resultant SO-TCSM symbols. Eavesdroppers cannot decode the confidential information delivered in the legitimate link, as they do not know the legitimate CSI and, hence, have no basis to acquire the transmitter's mapping pattern of the moment. Moreover, the SO-TCSM symbols are then compensated to maximise their Euclidean distances, which further improves the legitimate receiver's decoding performance while enhancing the PLS. However, the compensation results in high transmit power when the legitimate channels are in deep fades. To boost the energy efficiency, a constraint is set to limit the transmit power. Under this constraint, the SO-TCSM performance is investigated in terms of bit error rate and transmit power consumption. The outcome of these investigations substantiates that, compared to conventional TCSM designs, our SO-TCSM achieves better performance in the legitimate link with lower decoding complexity.
\end{abstract}

Index Terms-Physical layer security (PLS), spatial modulation (SM), trellis coded modulation (TCM), trellis coded spatialmodulation (TCSM).

\section{INTRODUCTION}

Since the communication security model was initiated by Shannon [1] and the wiretap channel model was conceived by Wyner [2], the physical layer security (PLS) has been developed in the aspects of information-theoretic study and communication protocol design [3], [4]. In the information-theoretic study, the secrecy capacity has been formulated in a variety of wiretap models, such as wireless fading channels [5], [6], multi-antenna configurations [7], [8] and broadcast systems [9]. In the secure communication protocol design, various techniques, e.g., beamforming [10], [11], relaying [12], and precoding [13], have been redeveloped to enhance the PLS for modern and future communication systems including the fifth generation (5G) mobile networks [14], full-duplex transmissions [15], deviceto-device communications [16], massive connectivity [17] and cognitive radio [18].

The majority of PLS techniques exploit multi-antenna transmissions to jam eavesdroppers' wiretapping while improving desired receivers' performance [19]. As one of the promising multi-antenna transmissions, spatial modulation (SM) [20]-[22] has been exploited for the PLS purpose as well. In particular, thanks to its special mapping of information, SM has been used to convert information in the implementation of secure communications [23]. Moreover, SM-aided jamming [24], [25],

M. Yin and B. Jiao are with the Department of Electronics, Peking University, Beijing 100871, China (e-mail: yinmx@pku.edu.cn, jiaobl@pku.edu.cn).

Y. Yang (corresponding author) is with the School of Engineering, University of Lincoln, Lincoln LN6 7TS, U.K. (e-mail: yyang@lincoln.ac.uk). precoding [26], [27] and artificial noise [28], [29] have been introduced to achieve the PLS under the assumption that the transmitter knows the channel state information (CSI) of both legitimate link and wiretapping link. In practice, the transmitter can always get the CSI of the link spanning from itself to the desired receiver without any feedback signalling in time division duplexing (TDD) systems. However, it is impractical to assume the transmitter is capable of getting the wiretapping CSI or its statistics if the eavesdropper is a non-authorised subscriber. Actually, this assumption is resulted from the aforementioned CSIbased PLS techniques which utilise the random characteristics of wireless channels to degrade the wiretapping link.

To release the assumption that the transmitter knows the wiretapping CSI, we attempt to develop PLS solutions based on the random characteristics of information sources rather than those of wireless channels. In general, there are two straightforward strategies to exploit the characteristics of information sources in the PLS implementation: (i) The desired receiver knows the bit-to-symbol mapping pattern in the legitimate link but eavesdroppers do not; and (ii) the free Euclidean distance between the constellation points received by the desired receiver is larger than those received by eavesdroppers. In [30], we proposed an information-source-based PLS solution in terms of the first strategy, which varies the SM patterns of the bitto-symbol mapping and the transmit antenna (TA) selection to degrade eavesdroppers' detection performance instead of their channel quality. This solution leads to a plainer transmission medium and provides more freedom for the communication protocol design, specially in ad-hoc networks. In this work, we integrate both strategies to propose a novel trellis coded SM (TCSM) for the PLS purpose, referred to as securityoriented TCSM (SO-TCSM), which not only varies the SM mapping patterns but also optimises the free Euclidean distance in the resultant constellation received by the desired receiver to improve the legitimate link performance while enhancing the PLS.

The main idea of trellis coded modulation (TCM) is to achieve maximum free Euclidean distance in the constellation of amplitude/phase modulation (APM) together with maximum binary Hamming distance in the channel coding [31]. In [32], the concept of TCM is applied to the TA index mapping in SM for the purpose of reducing the effect of correlated channels on the SM performance, while leaving the APM mapping uncoded. Then, a turbo TCSM scheme is proposed in [33], where the information bits mapped by the TA index are turbo-trellis-coded, with interleaving and puncturing, to achieve a coding gain over the TCSM scheme in [32], but the information bits mapped by the APM symbols are still not encoded therein. Moreover, the idea of TCM has been exploited to process the TA index mapping for generalised SM as well [34], [35]. In [36], SM with trellis coding (SM-TC) is proposed to exploit trellis encoding 
directly for both APM mapping and TA selection in the SM. Subsequently, SM with turbo trellis coding is proposed in [37] to increase the coding gain, where the information bits mapped by APM symbols and TA indices are encoded by the same encoder as in [33]. As a further advance, the computational complexity of code search for this scheme is reduced in [38] through the use of symbol-based extrinsic information transfer charts with a relaxation. In addition, the SM design is optimised in [39] by using machine learning to solve the data-driven prediction in the TA selection and power allocation.

In a SM system with $M$ TAs and $K$-point APM, there are $M$ scaled and rotated $K$-point APM constellations resulted from various fading channels pertaining to multiple TAs. The scaling and rotation are variable thanks to the randomness of wireless channels. This is a distinctive attribute in SM, but has not been taken into account by previous works on SM with TCM. Motivated by this, our proposed SO-TCSM scheme designs the TCSM mapping based on the various scaling and rotation to achieve maximum free Euclidean distance in the resultant constellation together with maximum binary Hamming distance in the trellis encoding, while exploiting the randomness of the scaling and rotation to provide PLS. Furthermore, the SO-TCSM symbols are compensated to form an optimal constellation at the desired receiver, which not only improves the desired receiver's detection performance but also reduces its detection complexity. However, the compensation will result in high transmit power if the activated channel is in a deep fade. To increase the energy efficiency, a constraint is set to control the transmit power. The impact of this transmit power constraint on the SO-TCSM performance is investigated, which substantiates the validity and robustness of our proposed SO-TCSM design.

In particular, our main contributions in this paper are threefold:

- Security Solution - A novel transmission strategy, referred to as SO-TCSM, is proposed to enhance the PLS through varying SM mapping patterns together with maximising the free Euclidean distance in the resultant SM constellation at desired receiver.

- Detection Improvement - With lower complexity in the desired receiver's detection, the proposed SO-TCSM scheme achieves better performance in the legitimate link than the SM-TC scheme of [36].

- Energy Efficiency - To reduce the power consumption, two transmission protocols for our SO-TCSM design are conceived under a constraint on the transmit power, and their performance has been investigated.

The remainder of this paper is organized as follows. Firstly, the wiretap channel model and a paradigm of the proposed SOTCSM design are presented in Section II. Subsequently, Section III illustrates the SO-TCSM scheme through the general design criteria and two examples, based on which its performance is evaluated in terms of bit error rate (BER). To further improve the energy efficiency, a constraint on the transmit power is set and its impact on the SO-TCSM performance is investigated in Section IV. Section V considers the application of the SOTCSM in more practical scenarios, where the legitimate link is degraded by estimated CSI and the wiretapping channel is

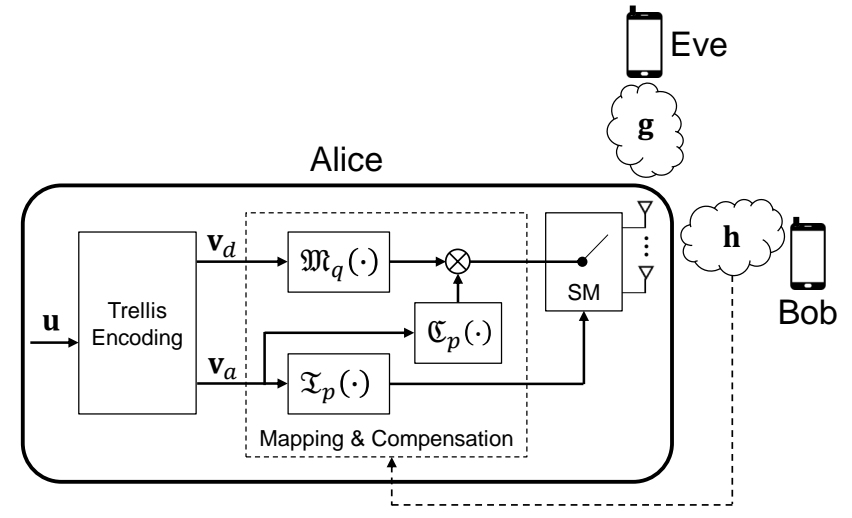

Fig. 1. Channel model of a SO-TCSM system.

highly correlated with the legitimate channel. Finally, Section VI concludes this paper and offers an insight of future research directions.

Throughout this paper, the following mathematical notations are used: Boldface uppercase and lowercase letters denote matrices and vectors, respectively. A calligraphic uppercase letter, e.g., $\mathcal{O}$, denotes a signal set, and the $i^{\text {th }}$ element in this set is denoted by $\mathcal{O}(i)$. The transpose, conjugate and modulus operators are denoted by $(\cdot)^{\mathrm{T}},(\cdot)^{*}$ and $|\cdot|$, respectively. Moreover, $M$ ! denotes the factorial of a non-negative integer $M$, and $\mathfrak{D}(\cdot)$ denotes the binary-to-decimal converter. In addition, $\operatorname{Pr}\{\cdot\}$ denotes the probability of an event and $\mathcal{E}\{\cdot\}$ represents the expectation (mean) operator. The complex normal distribution with mean $\mu$ and variance $\sigma^{2}$ is denoted by $\mathcal{C N}\left(\mu, \sigma^{2}\right)$.

\section{Security-Oriented Trellis Coded SPATIAL-MODULATION}

In this section, the SO-TCSM is proposed for a multi-antenna wiretap channel and a paradigm is presented to illustrate the SO-TCSM design.

\section{A. Channel Model}

Consider a multi-antenna wiretap channel model shown in Fig. 1, where the legitimate transmitter Alice, with $M$ TAs, delivers confidential messages to the single-antenna desired receiver Bob. A single-antenna eavesdropper Eve wiretaps Alice's transmitting and attempts to unveil the confidential messages.

The legitimate channel spanning from Alice to Bob and the wiretapping channel, i.e., from Alice to Eve, are denoted by $1 \times M$ vectors $\mathbf{h}=\left[h_{0}, h_{1}, \cdots, h_{M-1}\right]$ and $\mathbf{g}=$ $\left[g_{0}, g_{1}, \cdots, g_{M-1}\right]$, respectively, where the entries $h_{m}$ and $g_{m}$ represent the channels' coefficients from Alice's TA $m$ to Bob and Eve, respectively, $m=0,1, \cdots, M-1$. All the channels' coefficients are independent and identically distributed with complex Gaussian distribution of zero mean and variance $\sigma_{H}^{2}$, i.e., $h_{m}, g_{m} \sim \mathcal{C N}\left(0, \sigma_{H}^{2}\right)$.

At Alice, the original information bit sequence $\mathbf{u}$ is encoded by a trellis encoder and the output is divided into two sequences, i.e., the antenna bit sequence $\mathbf{v}_{a}$ and the radiated bit sequence $\mathbf{v}_{d}$. In a SM transmission, $\mathbf{v}_{d}$ is mapped onto a symbol and radiated by the antenna selected by $\mathbf{v}_{a}$. 
To achieve PLS while enhancing the SM performance, Alice reckons on the legitimate CSI $\mathbf{h}$ to determine the TA index mapping pattern $\mathfrak{T}_{p}(\cdot)$ together with the compensation pattern $\mathfrak{C}_{p}(\cdot)$ as well as the radiated bit-to-symbol mapping pattern $\mathfrak{M}_{q}(\cdot), p=1,2, \cdots, P, q=1,2, \cdots, Q$, where $P$ is the number of TA index mapping patterns, determining the number of compensation patterns, and $Q$ is the number of available bit-to-symbol mapping patterns.

The mapping patterns $\mathfrak{T}_{p}(\cdot)$ and $\mathfrak{M}_{q}(\cdot)$ together with the compensation pattern $\mathfrak{C}_{p}(\cdot)$ in the SO-TCSM design are detailed in the following paradigm.

\section{B. A SO-TCSM Paradigm}

Herein, the SO-TCSM scheme is illustrated by a paradigm where Alice sets $M=4$ TAs and adopts quadrature phase-shift keying (QPSK) for the SM transmissions. Thus, the channels from Alice to Bob and Eve are $\mathbf{h}=\left[h_{0}, h_{1}, h_{2}, h_{3}\right]$ and $\mathbf{g}=$ $\left[g_{0}, g_{1}, g_{2}, g_{3}\right]$, respectively. The QPSK symbol set is denoted by $\mathcal{F}=\left\{s_{0}, s_{1}, s_{2}, s_{3}\right\}$ and the symbols $s_{k}=e^{j k \pi / 2}, k=$ $0,1,2,3$. In this paradigm, both $\mathbf{v}_{a}$ and $\mathbf{v}_{d}$ are $1 \times 2$ vectors.

At Bob, the SM-QPSK constellation is composed of four scaled and rotated QPSK constellations, i.e., there are 16 symbols $h_{m} s_{k}$, where $m, k=0,1,2,3$. Apparently, the optimal constellation to locate these 16 symbols is the one for 16quadrature amplitude modulation (16QAM), given by Fig. 2 with a real number $\alpha>0$ used to adjust the average symbol energy of this constellation, which is the target constellation for this paradigm design to form. The 16QAM symbol set is denoted by $\mathcal{O}=\{\mathcal{O}(1), \mathcal{O}(2), \cdots, \mathcal{O}(16)\}$, and these symbols are mapped by 4-bit sequences $\mathbf{v}=\left[\mathbf{v}_{a}, \mathbf{v}_{d}\right]$ via natural binarycoded decimal of their indices.

These 16QAM symbols are divided into four subsets marked in different shapes and the $\hat{m}^{\text {th }}$ subset, pertaining to TA $\sharp \hat{m}$, is denoted by $\mathcal{H}_{\sharp \hat{m}}=\left\{\mathcal{H}_{\sharp \hat{m}}(1), \mathcal{H}_{\sharp \hat{m}}(2), \mathcal{H}_{\sharp \hat{m}}(3), \mathcal{H}_{\sharp \hat{m}}(4)\right\}=$ $\{\mathcal{O}(4 \hat{m}-3), \mathcal{O}(4 \hat{m}-2), \mathcal{O}(4 \hat{m}-1), \mathcal{O}(4 \hat{m})\}, \hat{m}=1,2,3,4$. As is shown in the figure, these four subsets are indexed by the two most significant bits (MSBs), i.e., $\mathbf{v}_{a}$. In terms of the TCM concept, the inter-subset Euclidean distance for the first MSB is larger than that for the second MSB, which guarantees that the former bit achieves better BER performance than the latter one. Within each subset, the third MSB and the least significant bit (LSB), i.e., $\mathbf{v}_{d}$, are mapped onto the QPSK symbols transmitted by each TA. The intra-subset Euclidean distance for the third MSB is $\sqrt{2}$ times that for the LSB, which is designed in terms of the TCM concept as well. That is, the two MSBs and the two LSBs are designed separately due to the subset partitioning based on the four various fading channels in our SO-TCSM paradigm, which is different from the MSB-to-LSB design in the traditional TCM.

In detail, Alice formats the SO-TCSM signals through three steps.

\section{Step 1 - Set the TA index mapping pattern $\mathfrak{T}_{p}(\cdot)$}

The TA index mapping pattern is selected according to the order of legitimate channels' modulus values. The modulus of the channel spanning from Alice's TA $m$ to Bob is $\left|h_{m}\right|, m=$ $0,1,2,3$, and these modulus values are sorted in an increasing order. The number of all possible orders is $P=4 !=24$.

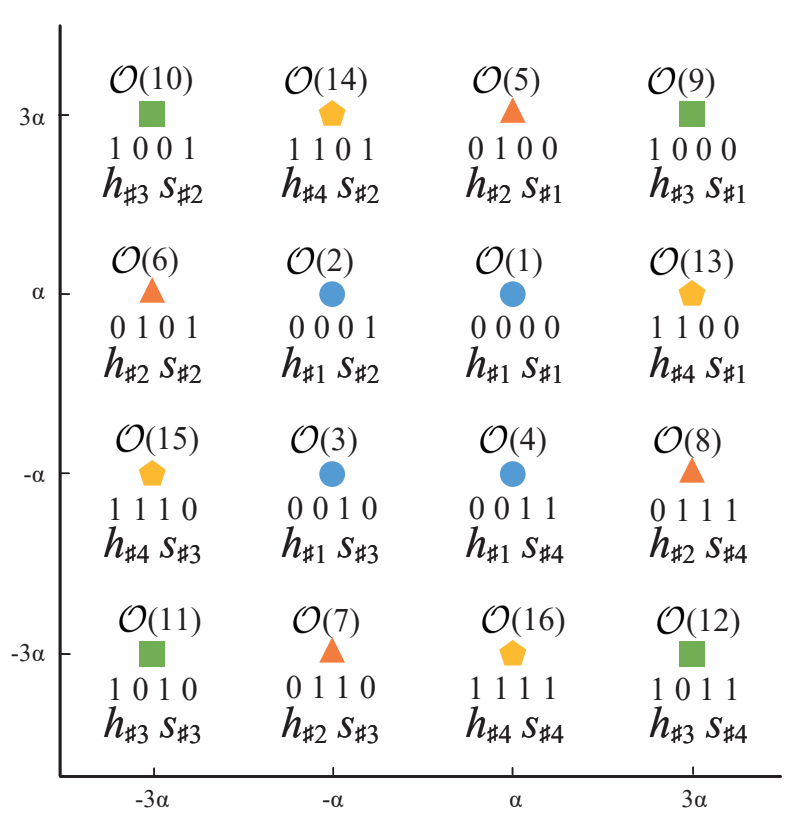

Fig. 2. Target constellation of the SO-TCSM paradigm.

In this step, the original index of a TA, denoted by $m \in\{0,1,2,3\}$, is replaced by the ordered index $\sharp \hat{m} \in$ $\{\sharp 1, \sharp 2, \sharp 3, \sharp 4\}$, according to the order of the symbol modulus values for the four subsets in Fig. 2. For the SO-TCSM target constellation, all the symbols' modulus values in a subset are the same, i.e., $\left|\mathcal{H}_{\sharp \hat{m}}(1)\right|=\left|\mathcal{H}_{\sharp \hat{m}}(2)\right|=\left|\mathcal{H}_{\sharp \hat{m}}(3)\right|=\left|\mathcal{H}_{\sharp \hat{m}}(4)\right|$. Therefore, the order of the symbol modulus values for the four subsets can be simplified as the order of the first symbol modulus in these subsets, i.e., $\left|\mathcal{H}_{\sharp 1}(1)\right|<\left|\mathcal{H}_{\sharp 2}(1)\right|=\left|\mathcal{H}_{\sharp 4}(1)\right|<$ $\left|\mathcal{H}_{\sharp 3}(1)\right|$, which sorts the subset order as $\sharp 1, \sharp 2, \sharp 4, \sharp 3$.

As such, the TAs pertaining to the smallest, the second smallest, the third smallest, and the largest channel modulus values are indexed by $\sharp 1, \sharp 2, \sharp 4$, and $\sharp 3$, respectively. The order of their channel modulus values can be expressed as $\left|h_{\sharp 1}\right| \leqslant\left|h_{\sharp 2}\right| \leqslant\left|h_{\sharp 4}\right| \leqslant\left|h_{\sharp 3}\right|$. Since the symbols in the subset $\mathcal{H}_{\sharp \hat{m}}$ are transmitted from TA $\sharp \hat{m}$, the difference is compensated by $\left|\mathcal{H}_{\sharp \hat{m}}(1)\right| /\left|h_{\sharp \hat{m}}\right|$. This index replacement minimises the difference between $\left|h_{\sharp \hat{m}}\right|$ and $\left|\mathcal{H}_{\sharp \hat{m}}(1)\right|$, thus leading to the minimum compensation power.

The number of TA index mapping patterns is equal to that of all possible orders, i.e., $P=24$. Without loss of generality, the mapping of antenna bits $\mathbf{v}_{a}$ onto an ordered TA index in the $p^{\text {th }}$ TA index mapping pattern, $p=1,2, \cdots, 24$, denoted by $\mathfrak{T}_{p}\left(\mathbf{v}_{a}\right)$, is expressed as $\mathfrak{T}_{p}(00)=\sharp 1, \mathfrak{T}_{p}(01)=\sharp 2, \mathfrak{T}_{p}(10)=$ $\sharp 3, \mathfrak{T}_{p}(11)=\sharp 4$. Based on the determined TA index mapping pattern, Alice activates the ordered TA subject to $\mathbf{v}_{a}$.

\section{Step 2 - Set the QPSK mapping pattern $\mathfrak{M}_{q}(\cdot)$}

The QPSK mapping pattern is denoted by $\sharp \hat{k} \rightarrow k$, where $\sharp \hat{k} \in\{\sharp 1, \sharp 2, \sharp 3, \sharp 4\}$ stands for the ordered index of a QPSK symbol and $k \in\{0,1,2,3\}$ is the natural index of a QPSK symbol.

As given by Fig. 3, the QPSK mapping pattern is selected according to the activated channel $h_{\sharp \hat{m}}$, and the number of QPSK mapping patterns is $Q=4$. The $q^{\text {th }}$ mapping pattern $\mathfrak{M}_{q}(\cdot)$ 


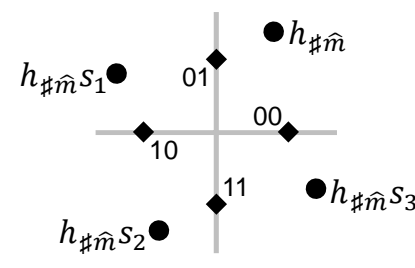

(a) $h_{\sharp \widehat{m}}$ in Quadrant I

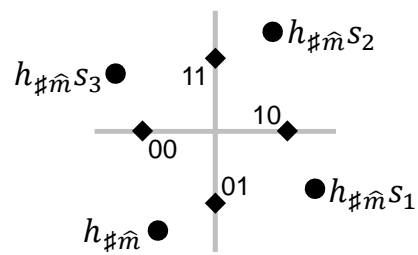

(c) $h_{\# \widehat{m}}$ in Quadrant III

- QPSK constellation point

- SM-QPSK constellation point for the channel $h_{\sharp \widehat{m}}$

Fig. 3. QPSK mapping patterns in the SO-TCSM paradigm under study.

bears upon the case that the channel fading $h_{\sharp \hat{m}}$ falls in the $q^{\text {th }}$ quadrant, $q=1,2,3,4$, and this pattern can be expressed as $\mathfrak{M}_{q}(00)=s_{\sharp 1}, \mathfrak{M}_{q}(01)=s_{\sharp 2}, \mathfrak{M}_{q}(10)=s_{\sharp 3}, \mathfrak{M}_{q}(11)=s_{\sharp 4}$, where $s_{\sharp \hat{k}}$ is the QPSK symbol that makes the SM-QPSK symbol $h_{\sharp \hat{m}} s_{\sharp \hat{k}}$ fall in the $\hat{k}^{\text {th }}$ quadrant, $\hat{k}=1,2,3,4$, given TA $\sharp \hat{m}$ is activated in Step 1 .

Based on the assigned QPSK mapping pattern, Alice maps the radiated bits $\mathbf{v}_{d}$ onto a QPSK symbol and transmits this symbol through the active TA.

Step 3 - Set the compensation pattern $\mathfrak{C}_{p}(\cdot)$

Through Step 1 and Step 2, Alice accomplishes the mapping of SM-QPSK symbols with 4 TAs, $h_{\sharp \hat{m}} s_{\sharp \hat{k}}, \hat{m}=1,2,3,4$, $\hat{k}=1,2,3,4$. To optimise Bob's detection performance, the transmitted symbols $s_{\sharp \hat{k}}, \hat{k}=1,2,3,4$, are compensated according to the active channel fading $h_{\sharp \hat{m}}$ to attain the target 16QAM constellation shown in Fig. 2, which is the resultant SO-TCSM constellation received by Bob.

Each SM-QPSK symbol is mapped by 4 bits; the first two bits are antenna bits, $\mathbf{v}_{a}$, mapped onto the fading channel $h_{\sharp \hat{m}}$ in Step 1 and the last two bits are radiated bits, $\mathbf{v}_{d}$, mapped onto the QPSK symbol $s_{\sharp \hat{k}}$ in Step 2. In detail, the symbols transmitted from TA $\sharp \hat{m}$ consist in the subset $\mathcal{H}_{\sharp \hat{m}}$, namely $\{\mathcal{O}(4 \hat{m}-3), \mathcal{O}(4 \hat{m}-2), \mathcal{O}(4 \hat{m}-1), \mathcal{O}(4 \hat{m})\}, \hat{m}=1,2,3,4$.

Since the compensations for the active channel fading $h_{\sharp \hat{m}}$ in the four quadrants are the same due to symmetry, the compensation pattern determined by the $p^{\text {th }}$ TA index mapping pattern can be calculated using $\mathfrak{C}_{p}\left(\mathbf{v}_{a}\right)=\mathcal{O}(4 \hat{m}-3) /\left(h_{\sharp \hat{m}} s_{\sharp 1}\right)$ given the activated TA $\sharp \hat{m}$ and the ordered index $\sharp \hat{m}=\mathfrak{T}\left(\mathbf{v}_{a}\right)$, where the symbol $\mathcal{O}(4 \hat{m}-3)$ is the one located in Quadrant I of the subset $\mathcal{H}_{\sharp \hat{m}}$, and $s_{\sharp 1}$ is the QPSK symbol that makes $h_{\sharp \hat{m}} s_{\sharp 1}$ fall in Quadrant I.

As a consequence, in an arbitrary SO-TCSM transmission interval, the received baseband signals at Bob and Eve are obtained by

$$
y_{\mathrm{B}}=h_{\mathfrak{T}_{p}\left(\mathbf{v}_{a}\right)} \mathfrak{C}_{p}\left(\mathbf{v}_{a}\right) \mathfrak{M}_{q}\left(\mathbf{v}_{d}\right)+z_{\mathrm{B}}
$$

and

$$
y_{\mathrm{E}}=g_{\mathfrak{T}_{p}\left(\mathbf{v}_{a}\right)} \mathfrak{C}_{p}\left(\mathbf{v}_{a}\right) \mathfrak{M}_{q}\left(\mathbf{v}_{d}\right)+z_{\mathrm{E}},
$$

respectively, where $y_{\mathrm{B}}$ and $z_{\mathrm{B}} \sim \mathcal{C N}\left(0, \sigma_{W}^{2}\right)$ are Bob's received signal and additive white Gaussian noise (AWGN). Meanwhile, $y_{\mathrm{E}}$ and $z_{\mathrm{E}} \sim \mathcal{C} \mathcal{N}\left(0, \sigma_{W}^{2}\right)$ are Eve's received signal and AWGN. The subscript " $\mathfrak{T}_{p}\left(\mathbf{v}_{a}\right)$ " denotes the index of the TA activated by antenna bits $\mathbf{v}_{a}$ according to the $p^{\text {th }}$ TA index mapping pattern $\mathfrak{T}_{p}(\cdot)$ set in Step 1. Thus, $h_{\mathfrak{T}_{p}\left(\mathbf{v}_{a}\right)}$ and $g_{\mathfrak{T}_{p}\left(\mathbf{v}_{a}\right)}$ are the channels' coefficients from Alice's active TA to Bob and Eve, respectively. Moreover, $\mathfrak{M}_{q}\left(\mathbf{v}_{d}\right)$ stands for Alice's transmitted QPSK symbol mapped by radiated bits $\mathbf{v}_{d}$ according to the $q^{\text {th }}$ QPSK mapping pattern $\mathfrak{M}_{q}(\cdot)$ set in Step 2. In addition, the transmitted symbol is compensated by $\mathfrak{C}_{p}\left(\mathbf{v}_{a}\right)$ according to the compensation pattern $\mathfrak{C}_{p}(\cdot)$ set in Step 3 .

\section{Detection}

Bob adopts the soft-output Viterbi algorithm (SOVA) to decode the SO-TCSM signals, by taking the Euclidean distance between the received symbol $y_{\mathrm{B}}$ and the expected symbol pertaining to the codeword as a branch metric. For the bit sequence $\mathbf{v}=\left[\mathbf{v}_{a}, \mathbf{v}_{d}\right]$, the Euclidean distance calculated by Bob is expressed as

$$
D_{\mathrm{B}}=\left|y_{\mathrm{B}}-h_{\mathfrak{T}_{p}\left(\mathbf{v}_{a}\right)} \mathfrak{C}_{p}\left(\mathbf{v}_{a}\right) \mathfrak{M}_{q}\left(\mathbf{v}_{d}\right)\right| .
$$

Based on the resultant SO-TCSM constellation given in Fig. 2, (3) can be simplified as

$$
D_{\mathrm{B}}=\left|y_{\mathrm{B}}-\mathcal{O}(\mathfrak{D}(\mathbf{v})+1)\right|,
$$

where $\mathfrak{D}(\cdot)$ denotes the binary-to-decimal converter. Hence, Bob's detection complexity using (4) is equivalent to that of a 16QAM constellation detection, which is much lower than the detection complexity of conventional TCSM schemes, e.g., the SM-TC in [36].

When it comes to Eve, she does not know the CSI of the legitimate link from Alice to Bob and, therefore, she cannot pick up the correct TA index mapping pattern or QPSK mapping pattern to decode the SO-TCSM signals. Moreover, she has no knowledge on the compensation pattern in the legitimate link. As such, Eve has to use her own mapping patterns for the soft decision and calculates the Euclidean distance for $\mathbf{v}$ as

$$
D_{\mathrm{E}}=\left|y_{\mathrm{E}}-g_{\mathfrak{T}_{\mathrm{E}}\left(\mathbf{v}_{a}\right)} \mathfrak{M}_{\mathrm{E}}\left(\mathbf{v}_{d}\right)\right|,
$$

where $\mathfrak{T}_{\mathrm{E}}(\cdot)$ and $\mathfrak{M}_{\mathrm{E}}(\cdot)$ are the TA index and QPSK symbol mapping patterns adopted by Eve, respectively. The probability that Eve correctly chooses the same mapping patterns as Alice dose is relatively low, which is $\operatorname{Pr}\left\{\mathfrak{T}_{\mathrm{E}}(\cdot)=\mathfrak{T}_{p}(\cdot), \mathfrak{M}_{\mathrm{E}}(\cdot)=\right.$ $\left.\mathfrak{M}_{q}(\cdot)\right\}=(1 / P)(1 / Q)=1 / 96$. Further, even if Eve could figure out the correct mapping patterns, the free Euclidean distance of its received constellation is smaller than that of Bob's, because Bob's received constellation has been optimised by the compensation pattern $\mathfrak{C}_{p}(\cdot)$. Thus, Eve's attempts to unveil Alice's transmissions will be nullified by our SO-TCSM scheme. 
TABLE I

2/4-RATE TRELLIS ENCODING.

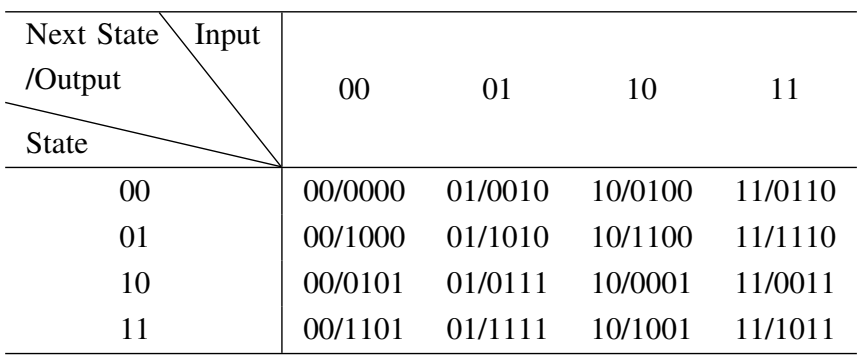

\section{Bit Error Rate}

The performance of our SO-TCSM scheme is validated by comparing Bob's and Eve's BERs. At Alice, the trellis encoder adopts the octal generator matrix $\left[\begin{array}{llll}0 & 3 & 0 & 1 \\ 1 & 0 & 2 & 0\end{array}\right]$ introduced by [40] with code rate $2 / 4$ and four states. As listed in Table I, the four transition outputs caused by the same input to the same trellis state are mapped onto the symbols in different subsets $\mathcal{H}_{\sharp \hat{m}}, \hat{m}=1,2,3,4$, specified by Fig. 2 . Moreover, all output branches from or to each trellis state are evenly spread across the Euclidean space. In the traditional TCM design [31], all branches from or to the same trellis state are expected to be mapped onto the symbols in a partition who has the maximum free Euclidean distances inside. However, in our SOTCSM paradigm, the partition of a 16QAM constellation into four subsets pertaining to various fading channels reduces the degrees of freedom in the set partitioning. Therefore, the classic trellis encoding structure designed for the traditional TCM is not the optimal one for the TCSM design.

In Fig. 4, the BER comparisons between Bob and Eve with our proposed SO-TCSM paradigm are reported versus the received signal-to-noise power ratio (SNR) $\sigma_{H}^{2} / \sigma_{W}^{2}$, where the BER of the SM-TC scheme proposed in [36] is also provided for the sake of comparison using the same trellis encoder in the same system configuration, i.e., SM through 4 TAs with QPSK. As is shown in this figure, Eve cannot successfully decode any information from her received signals, while Bob achieves better performance than the SM-TC scheme in [36]. The results substantiate that our SO-TCSM design not only enhances the PLS to prevent wiretapping but also exploits the TCM structure more efficiently in SM systems to ensure better decoding performance achieved by the desired receiver, in comparison to the SM-TC in [36]. The main reason behind this is that the impact of random channel fading on the resultant SM-QPSK constellation at the desired receiver has not been taken into account by the TCM design in [36], while we exploit the randomness of channel fading to achieve maximum free Euclidean distance along with maximum binary Hamming distance in the SO-TCSM design.

\section{Design CRiteria AND Instances}

\section{A. General SO-TCSM Design}

For the general case of the SO-TCSM design, consider the wiretap channel model in Fig. 1 with $M$ TAs at Alice who adopts $K$-point APM constellation, e.g., $K$-PSK or $K$-QAM

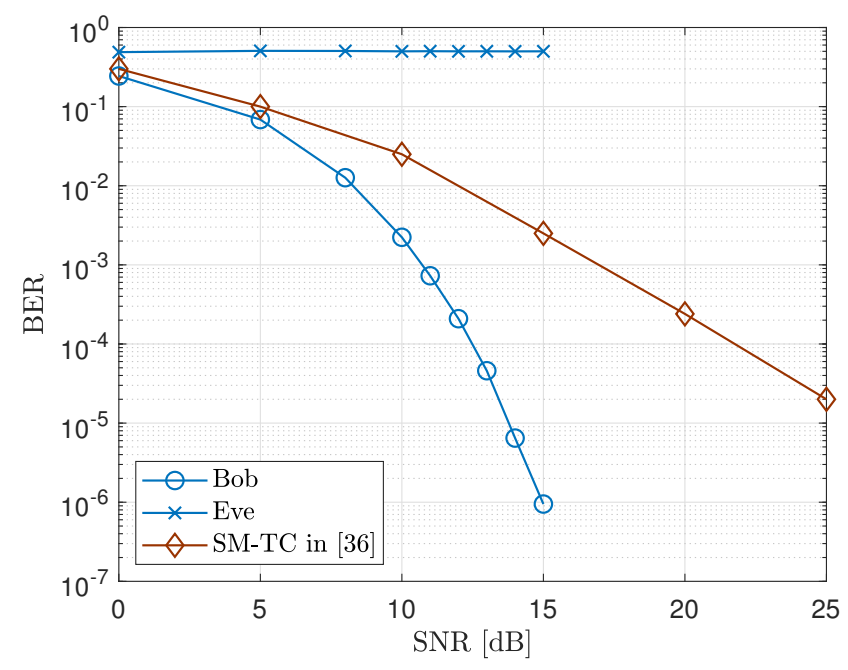

Fig. 4. BER performance of the SO-TCSM paradigm versus received SNR.

with the symbol set $\mathcal{F}=\left\{s_{0}, s_{1}, \cdots, s_{K-1}\right\}$. In an arbitrary SM transmission, the output of trellis encoding is a bit sequence $\mathbf{v}=\left[\mathbf{v}_{a}, \mathbf{v}_{d}\right]$ of length $N=\log _{2}(M K)$, where the vector $\mathbf{v}_{a}$ is composed of $\log _{2} M$ antenna bits and $\mathbf{v}_{d}$ consists of $\log _{2} K$ radiated bits.

With the SO-TCSM design, mapping patterns for $\mathbf{v}_{a}$ and $\mathbf{v}_{d}$ are determined by the legitimate CSI $\mathbf{h}=\left[h_{0}, h_{1}, \cdots, h_{M-1}\right]$ and the resultant constellation is shaped to maximise the minimum Euclidean distance through the following three steps.

Step 1 - Set a target constellation of $M K$ symbol points.

The target constellation is the resultant one received by Bob, which is composed of $M K$ symbol points, and all the points are uniformly distributed to leverage Bob's decoding performance. The symbol set in this constellation is denoted by $\mathcal{O}=\{\mathcal{O}(1), \mathcal{O}(2), \cdots, \mathcal{O}(M K)\}$ and the $N$-bit sequences $\mathbf{v}=\left[\mathbf{v}_{a}, \mathbf{v}_{d}\right]$ mapped onto these symbols are the natural binary-coded decimal values of their indices. These symbols are evenly divided into $M$ subsets pertaining to the delivery of $M$ TAs. The $\hat{m}^{\text {th }}$ subset is denoted by $\mathcal{H}_{\sharp \hat{m}}=$ $\left\{\mathcal{H}_{\sharp \hat{m}}(1), \mathcal{H}_{\sharp \hat{m}}(2), \cdots, \mathcal{H}_{\sharp \hat{m}}(K)\right\}, \hat{m}=1,2, \cdots, M$, which can be formatted by scaling and/or rotating the $K$-PSK/QAM constellation adopted by Alice.

We remark that, since the SO-TCSM target constellation consists of $M$ subsets that are the scaled and/or rotated versions of the same $K$-point constellation, the optimum $M K$-point constellation with the minimum error rate in AWGN channels, introduced in [41], is not always suitable for our target design.

At Bob, the average symbol energy of the target constellation is set to the same as that of Alice's $K$-PSK/QAM constellation, i.e.,

$$
\frac{1}{M K} \sum_{i=1}^{M K}|\mathcal{O}(i)|^{2}=\frac{\sigma_{H}^{2}}{K} \sum_{k=0}^{K-1}\left|s_{k}\right|^{2},
$$

where $\mathcal{O}(i)$ is the $i^{\text {th }}$ symbol in the $M K$-point constellation $\mathcal{O}, i=1,2, \cdots, M K$, and $s_{k}$ is the $k^{\text {th }}$ symbol in the $K$-point APM constellation, $k=0,1, \cdots, K-1$.

Step 2 - Set the TA index mapping pattern and the radiated bit-to-symbol mapping pattern. 
Basically, the TA index mapping pattern is determined by an increasing order of legitimate channels' modulus values, as described in the paradigm. The number of TA index mapping patterns is equal to that of all possible orders, i.e., $P=M$ !. The original index of a TA, $m \in\{0,1, \cdots, M-1\}$, is replaced by the ordered index $\sharp \hat{m} \in\{\sharp 1, \sharp 2, \cdots, \sharp M\}$, given an increasing order of the symbol modulus values for the $M$ subsets in the target constellation.

As the $M$ subsets in the SO-TCSM target constellation are the scaled and/or rotated versions of the same $K$-point constellation, all the symbols' modulus values in a subset are the same, i.e., $\left|\mathcal{H}_{\sharp \hat{m}}(1)\right|=\left|\mathcal{H}_{\sharp \hat{m}}(2)\right|=\cdots=\left|\mathcal{H}_{\sharp \hat{m}}(K)\right|$. Hence, the ascending order of the symbol modulus values for the $M$ subsets can be simplified as the ascending order of the first symbol modulus in these subsets, $\left|\mathcal{H}_{\sharp \hat{m}}(1)\right|$.

Then, the index replacement can be expressed as

$$
\sharp \hat{m} \rightarrow m: \operatorname{ord}\left(\left|\mathcal{H}_{\sharp \hat{m}}(1)\right|\right)=\operatorname{ord}\left(\left|h_{m}\right|\right),
$$

where $\operatorname{ord}(\cdot)$ denotes the order of an element in the ordered group. That is, the ordered index $\sharp \hat{m}$ is assigned to TA $m$ if the order of $\left|\mathcal{H}_{\sharp \hat{m}}(1)\right|$ in the ascending order of the symbol modulus values for the $M$ subsets is the same as the order of $\left|h_{m}\right|$ in the ascending order of the channels' modulus values.

This index replacement minimises the difference between $\left|h_{\sharp \hat{m}}\right|$ and $\left|\mathcal{H}_{\sharp \hat{m}}(1)\right|$, thus leading to the minimum compensation power $\left|\mathcal{H}_{\sharp \hat{m}}(1)\right| /\left|h_{\sharp \hat{m}}\right|$.

As such, the $K$ symbols in the subset $\mathcal{H}_{\sharp \hat{m}}$ are delivered through TA $\hat{m}$. In the $p^{\text {th }}$ pattern, $p=1,2, \cdots, P$, the mapping of the antenna bits $\mathbf{v}_{a}$ onto the TA index is expressed as $\mathfrak{T}_{p}\left(\mathbf{v}_{a}\right)=\sharp\left(\mathfrak{D}\left(\mathbf{v}_{a}\right)+1\right)$.

Upon the activation of the TA index, the radiated bit-tosymbol mapping pattern $\mathfrak{M}_{q}(\cdot), q=1,2, \cdots, Q$, is assigned according to the active channel $h_{\mathfrak{T}_{p}\left(\mathbf{v}_{a}\right)}$ and accommodates the spatially modulated $K$-PSK/QAM symbols $h_{\mathfrak{T}_{p}\left(\mathbf{v}_{a}\right)} s_{k}, k=$ $0,1, \cdots, K-1$, to the corresponding locations in the $\left(\mathfrak{D}\left(\mathbf{v}_{a}\right)+\right.$ $1)^{\text {th }}$ subset $\mathcal{H}_{\sharp\left(\mathcal{D}\left(\mathbf{v}_{a}\right)+1\right)}$ of the target constellation $\mathcal{O}$. The number of radiated bit-to-symbol mapping patterns is $Q=K$. In the $q^{\text {th }}$ radiated bit-to-symbol mapping pattern, the mapping of the radiated bits $\mathbf{v}_{d}$ onto a $K$-PSK/QAM symbol can be expressed as $\mathfrak{M}_{q}\left(\mathbf{v}_{d}\right)=s_{\sharp\left(\mathfrak{D}\left(\mathbf{v}_{d}\right)+1\right)}$, where $\sharp\left(\mathfrak{D}\left(\mathbf{v}_{d}\right)+1\right)$ is the ordered index of a $K$-PSK/QAM symbol.

Therefore, the total number of mapping patterns in the SOTCSM is $P Q=M ! K$.

Step 3 - Set the compensation pattern to achieve the target constellation.

For the purpose of optimising Euclidean distances between resultant SO-TCSM signals, the symbol $h_{\mathfrak{T}_{p}\left(\mathbf{v}_{a}\right)} \mathfrak{M}_{q}\left(\mathbf{v}_{d}\right)$ is compensated by $\mathfrak{C}_{p}\left(\mathbf{v}_{a}\right)=\left|\mathcal{H}_{\sharp\left(\mathcal{D}\left(\mathbf{v}_{a}\right)+1\right)}(1)\right| /\left|h_{\sharp\left(\mathcal{D}\left(\mathbf{v}_{a}\right)+1\right)}\right|$ to approach the corresponding point in the target constellation.

At Bob, the detection of the SO-TCSM signals is equivalent to the demodulation of the target constellation, which dramatically reduces Bob's calculation complexity. Using SOVA, the Euclidean distance for $\mathbf{v}=\left[\mathbf{v}_{a}, \mathbf{v}_{d}\right]$ is taken as a branch metric, calculated by

$$
D_{\mathrm{B}}=\left|y_{\mathrm{B}}-\mathcal{O}(\mathfrak{D}(\mathbf{v})+1)\right| .
$$

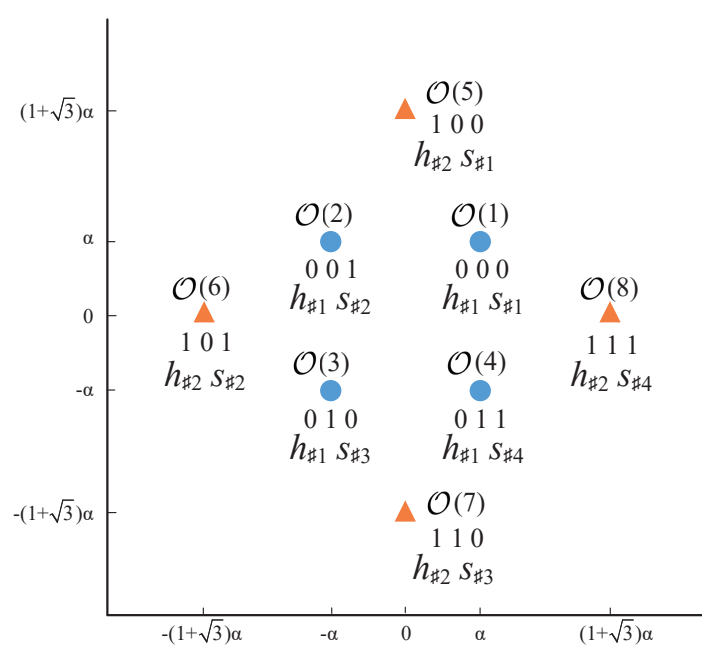

Fig. 5. Target constellation of the SO-TCSM with QPSK and 2 TAs.

Note that, although this Euclidean distance expression is the same as $(4), \mathcal{O}(\mathfrak{D}(\mathbf{v})+1)$ represents different symbols in different SM systems.

On the other hand, as Eve has no information on which mapping patterns are exploited over the legitimate link, she has to adopt her own mapping patterns to detect the received signals and will fail to reveal the confidential messages delivered from Alice.

\section{B. Two SO-TCSM Examples}

Since QPSK is one of the most popular modulation schemes used in current communication networks, e.g., DVB-S2 [42], LTE [43], etc., and the target constellation scale resulted from the SO-TCSM with QPSK is always manageable, herein we further exemplify the SO-TCSM scheme with the designs of 2 TAs and 8 TAs using QPSK. The QPSK symbols are denoted by $s_{k}=e^{j k \pi / 2}, k=0,1,2,3$. Note that, the SO-TCSM can be directly applied into the systems with other modulation schemes, based on the general 3-step design criteria presented in Section III-A.

1) $M=2$ TAs with QPSK: In this case, $\mathbf{v}_{a}$ contains 1 bit and $\mathbf{v}_{d}$ contains 2 bits. The target constellation is set in Fig. 5, where the real positive number $\alpha$ in the scales is used to align the average symbol energy of this constellation with that of QPSK. There are 2 subsets, i.e., the inner subset $\mathcal{H}_{\sharp 1}$ and the outer subset $\mathcal{H}_{\sharp 2}$. The TA with the closest channel fading to the subset $\mathcal{H}_{\sharp \hat{m}}$ is indexed by $\sharp \hat{m}, \hat{m}=1,2$.

The QPSK mapping patterns for the channel $h_{\sharp 1}$ are the same as those specified in Fig. 3. However, the QPSK mapping pattens for the channel $h_{\sharp 2}$ need to be set in a different manner that is given by Fig. 6, where the signal space is partitioned into 4 regions by 4 rays from the pole, and the $q^{\text {th }}$ region ranges from the ray of angle $(q-1) \pi / 2+\pi / 4$ to the one of angle $q \pi / 2+\pi / 4, q=1,2,3,4$. In detail, the $q^{\text {th }}$ QPSK mapping pattern, pertaining to the case that $h_{\sharp 2}$ falls in the $q^{\text {th }}$ region, can be expressed as $\mathfrak{M}_{q}\left(\mathbf{v}_{d}\right)=s_{\sharp \hat{k}}$, where $s_{\sharp \hat{k}}$ is the QPSK symbol that makes the SM-QPSK symbol $h_{\sharp 2} s_{\sharp \hat{k}}$ fall in the 


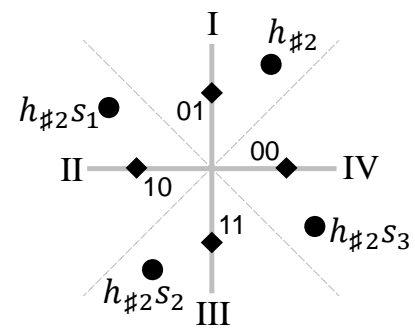

(a) $h_{\# 2}$ in Region I

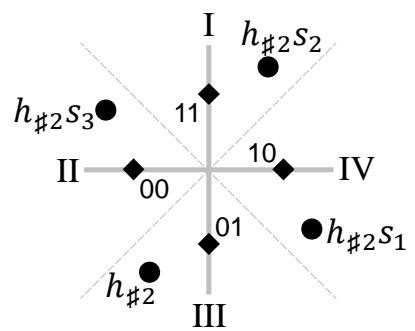

(c) $h_{\# 2}$ in Region III

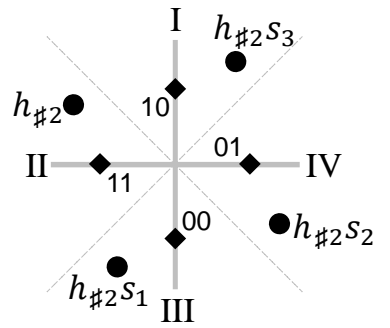

(b) $h_{\# 2}$ in Region II

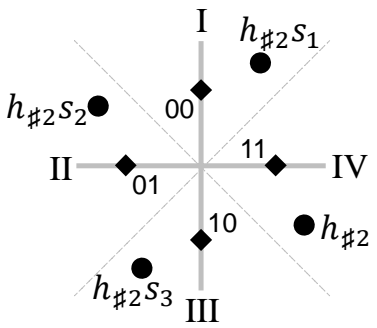

(d) $h_{\# 2}$ in Region IV

- QPSK constellation point

- SM-QPSK constellation point for the channel $h_{\sharp 2}$

Fig. 6. QPSK mapping patterns for the channel $h_{\sharp 2}$ in the example of $M=2$ TAs.

$\hat{k}^{\text {th }}$ region, $\hat{k}=1,2,3,4$. Elaborating slightly further, we have $\hat{k}=\mathfrak{D}\left(\mathbf{v}_{d}\right)+1$ in this mapping. Then, the compensation is made up through $\mathfrak{C}_{p}\left(\mathbf{v}_{a}\right)=\mathcal{O}(4 \hat{m}-3) /\left(h_{\sharp \hat{m}} s_{\sharp 1}\right)$ given the activated TA $\sharp \hat{m}, \hat{m} \in\{1,2\}$, where $s_{\sharp 1}$ is the QPSK symbol that makes $h_{\sharp 1} s_{\sharp 1}$ fall in Quadrant I if TA $\sharp 1$ is activated or the one that makes $h_{\sharp 2} s_{\sharp 1}$ fall in the first region indicated by Fig. 6 if TA $\sharp 2$ is activated.

2) $M=8 T$ TAs with QPSK: In this case, $\mathbf{v}_{a}$ contains 3 bits and $\mathbf{v}_{d}$ contains 2 bits. The target constellation is set in Fig. 7, where the eight subsets $\mathcal{H}_{\sharp \hat{m}}, \hat{m}=1,2, \cdots, 8$, in various shapes are paired with the 8 TAs. The TA having the closest channel fading to the subset $\mathcal{H}_{\sharp \hat{m}}$ is indexed by $\sharp \hat{m}, \hat{m}=1,2, \cdots, 8$. Subsequently, the QPSK mapping patterns given an activated channel are the same as those specified in Fig. 3, and the compensation is made by $\mathfrak{C}_{p}\left(\mathbf{v}_{a}\right)=\mathcal{O}(4 \hat{m}-3) /\left(h_{\sharp \hat{m}} s_{\sharp 1}\right)$ if TA $\sharp \hat{m}$ is activated, $\hat{m} \in\{1,2,3,4,5,6,7,8\}$, where $s_{\sharp 1}$ is the QPSK symbol that makes $h_{\sharp \hat{m}} s_{\sharp 1}$ fall in Quadrant I.

The BER comparisons of these two examples are shown in Fig. 8, where the case of $M=4$ TAs is also plotted. For the cases of $M=2$ and $M=4$, Alice utilises 2/3-rate and 2/4-rate trellis encoders, respectively, with the 4-state generator matrices $\mathbf{A}_{2}=\left[\begin{array}{lll}2 & 0 & 1 \\ 1 & 3 & 0\end{array}\right]$ and $\mathbf{A}_{4}=\left[\begin{array}{llll}0 & 3 & 0 & 1 \\ 1 & 0 & 2 & 0\end{array}\right]$. Without loss of generality, the SO-TCSM signals are assumed to be sampled at the Nyquist rate and, thus, the transmission rates in the cases of $M=2$ and $M=4$ are the same, i.e., both equal to $2 \mathrm{bits} / \mathrm{sec} / \mathrm{Hz}$. For the case of $M=8,3 / 5$-rate trellis encoding is adopted at Alice with the 4-state generator matrix $\mathbf{A}_{8}=\left[\begin{array}{lllll}1 & 0 & 0 & 0 & 0 \\ 0 & 0 & 3 & 0 & 1 \\ 0 & 1 & 0 & 2 & 0\end{array}\right]$ to achieve a higher data rate, i.e., $3 \mathrm{bits} / \mathrm{sec} / \mathrm{Hz}$. Note that these generator matrices are not optimised trellis encoding structures for the SO-TCSM design to achieve optimal BER performance over

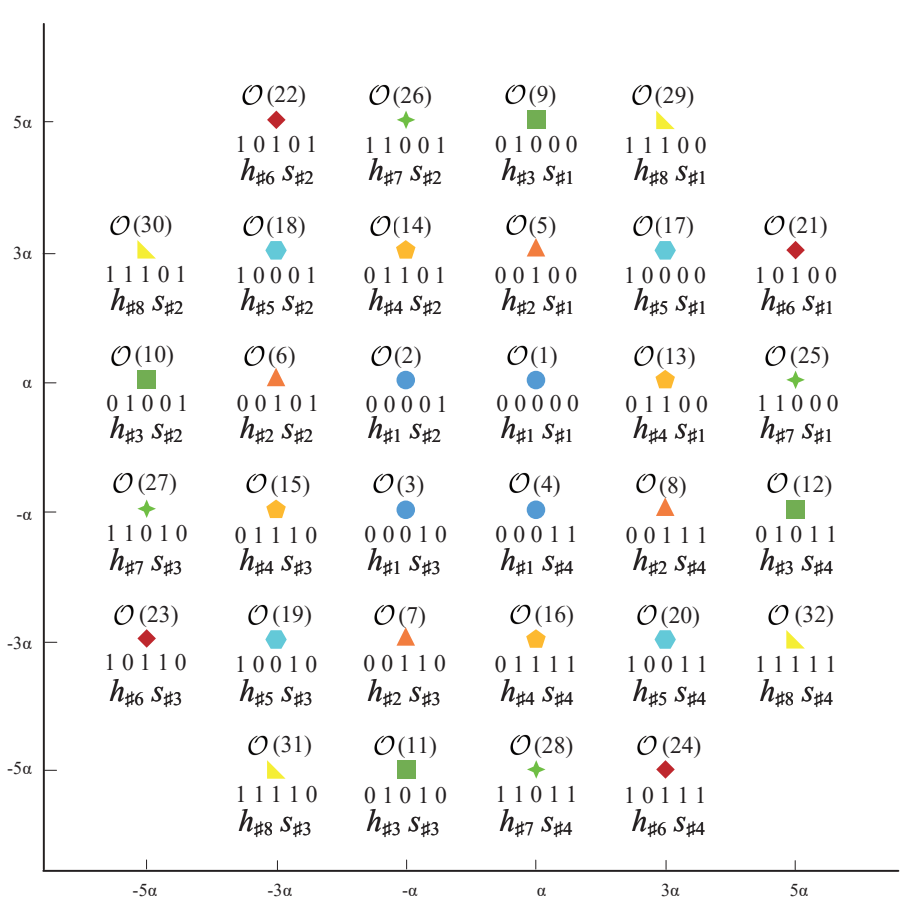

Fig. 7. Target constellation of the SO-TCSM with QPSK and 8 TAs.

the legitimate link. The matrix $\mathbf{A}_{4}$ used in the case of $M=4$ is the one who generates a classic encoding structure for the traditional TCM [40], while the matrices $\mathbf{A}_{2}$ and $\mathbf{A}_{8}$ are simply transferred from $\mathbf{A}_{4}$ for a brief comparison of the SO-TCSM performance in various multi-antenna configurations.

As is shown in Fig. 8, Eve's BER is always around 0.5 for different $M$, which implies that Eve cannot unveil any confidential messages delivered over the legitimate link relying on our SO-TCSM design. The information capacity of a binary symmetric channel with BER $p_{e}$ is $1-\mathrm{H}_{\mathrm{b}}\left(p_{e}\right)$, where

$$
\mathrm{H}_{\mathrm{b}}\left(p_{e}\right)=-p_{e} \log _{2} p_{e}-\left(1-p_{e}\right) \log _{2}\left(1-p_{e}\right)
$$

is the binary entropy function [44]. Since the BER $p_{e}=0.5$, Eve's information capacity is 0 . In other words, the security is guaranteed via the introduction of randomness in the information mapping and shaping. Additionally, the PLS offered by the SO-TCSM is not influenced by the antenna scale and can be guaranteed by even the simplest multi-antenna configuration. Moreover, we may notice that Bob's BER increases with the increase in the number of TAs, $M$. The main reason behind this is that, to maintain the same average symbol energy of the target constellation, specified by (6), the Euclidean distances between the symbols in the target constellation declines as $M$ increases.

\section{TRAnsmit Power CONSTRAints}

With the SO-TCSM, the transmit power in an arbitrary transmission interval is denoted by $P_{t}=\left|\mathfrak{C}_{p}\left(\mathbf{v}_{a}\right) \mathfrak{M}_{q}\left(\mathbf{v}_{d}\right)\right|^{2}=$ $\left|\mathfrak{C}_{p}\left(\mathbf{v}_{a}\right)\right|^{2}$, since $\mathfrak{M}_{q}\left(\mathbf{v}_{d}\right)$ is a QPSK symbol of unit energy. Apparently, the transmit power is determined by the compensation $\mathfrak{C}_{p}\left(\mathbf{v}_{a}\right)=\mathcal{O}(4 \hat{m}-3) /\left(h_{\sharp \hat{m}} s_{\sharp 1}\right), \hat{m}=1,2, \cdots, M$, and therefore affected by the instantaneous CSI pertaining to the 


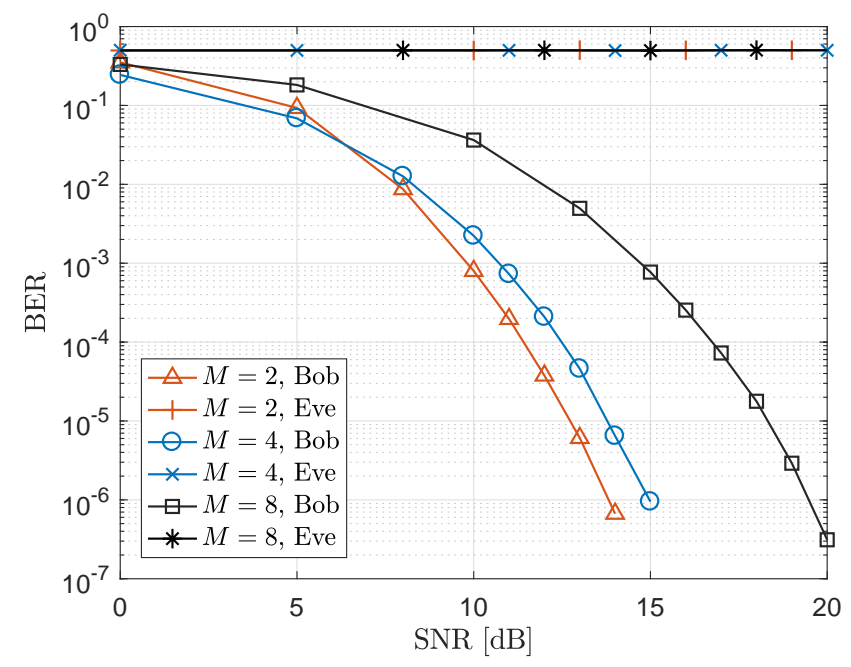

Fig. 8. BER comparisons between Bob and Eve in the SO-TCSM systems with QPSK and $M=2,4,8$ TAs.

activated TA, $h_{\mathfrak{T}_{p}\left(\mathbf{v}_{a}\right)}$, where $\mathfrak{T}_{p}\left(\mathbf{v}_{a}\right)=\sharp \hat{m}$. If the activated channel is in a deep fade, the transmit power will be very high. To reduce the transmit power in this case, a constraint on the transmit power, $\beta$, has to be set based on Alice's radio module or the energy efficiency requirement [45]. For example, typical transmit power is $20 \mathrm{~W}$ for the base station (BS) in a macro-cell, ranges from $0.2 \mathrm{~W}$ to $1 \mathrm{~W}$ for the base station in a pico-cell, and is lower than $0.2 \mathrm{~W}$ for the base station in a femtocell.

In the following, the impact of this transmit power constraint on our SO-TCSM performance is evaluated. We remark that, the transmit power of a QPSK symbol is deemed a benchmark for the unit power, namely 1 milliwatt $(\mathrm{mW})$. On this basis, the transmit power is measured and the constraint is set much lower than those typical values.

\section{A. PDF of Transmit Power without Constraint}

To begin with, we calculate the probability density function (PDF) of the transmit power $P_{t}$ without any constraint. Based on the criteria for Step 1 of the design paradigm in Section II-B and (7) in Step 2 of the general design, the order of the first symbol modulus in a subset, $\left|\mathcal{H}_{\sharp \hat{m}}(1)\right|$, is the same as that of the indexed channel modulus $\left|h_{\sharp m}\right|$. For example, $\left|\mathcal{H}_{\sharp \hat{m}}(1)\right|=|\mathcal{O}(4 \hat{m}-3)|$, $\hat{m}=1,2,3,4$, in the paradigm presented by Fig. 2, where the indexed channel modulus $\left|h_{\sharp 1}\right| \leqslant\left|h_{\sharp 2}\right| \leqslant\left|h_{\sharp 4}\right| \leqslant\left|h_{\sharp 3}\right|$.

In terms of the ascending order, the $l^{\text {th }}$ smallest channel fading is denoted by $\left|h_{(l)}\right|$, and the $l^{\text {th }}$ smallest modulus among $\left|\mathcal{H}_{\sharp \hat{m}}(1)\right|, \hat{m}=1,2, \cdots, M$, is denoted by $\left|\mathcal{H}_{(l)}(1)\right|$. The compensation for the difference between $\left|h_{(l)}\right|$ and $\left|\mathcal{H}_{(l)}(1)\right|$ is expressed as $\left|\mathfrak{C}_{(l)}\right|=\left|\mathcal{H}_{(l)}(1)\right| /\left|h_{(l)}\right|, l=1,2, \cdots, M$.

According to the order statistics [46], the PDF of $\left|h_{(l)}\right|$ is obtained by

$$
f_{l}(x)=\frac{M !\left[F_{\mathrm{R}}(x)\right]^{l-1}\left[1-F_{\mathrm{R}}(x)\right]^{M-l} f_{\mathrm{R}}(x)}{(l-1) !(M-l) !},
$$

where

$$
f_{\mathrm{R}}(x)=\frac{x}{\sigma_{H}^{2} / 2} \exp \left(-x^{2} / \sigma_{H}^{2}\right)
$$

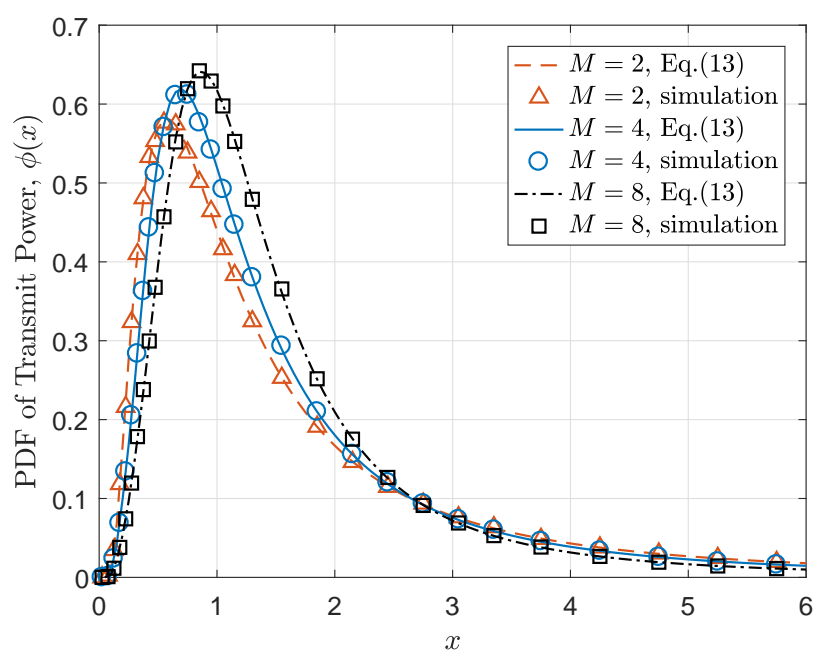

Fig. 9. The PDF of transmit power without any constraint, $\phi(x)$, in the SOTCSM systems with QPSK and $M=2,4,8$ TAs.

is the PDF of $\left|h_{m}\right|$, i.e., a Rayleigh distribution, and $F_{\mathrm{R}}(x)=$ $\int_{0}^{x} f_{\mathrm{R}}(x) d x$ is the cumulative distribution function of $\left|h_{m}\right|$.

Given the compensation $\left|\mathfrak{C}_{(l)}\right|=\left|\mathcal{H}_{(l)}(1)\right| /\left|h_{(l)}\right|$, the PDF of $\left|\mathfrak{C}_{(l)}\right|$ is derived by

$$
\begin{aligned}
\nu_{l}(x) & =f_{l}\left(\frac{\left|\mathcal{H}_{(l)}(1)\right|}{x}\right)\left|\frac{d \frac{\left|\mathcal{H}_{(l)}(1)\right|}{x}}{d x}\right| \\
& =f_{l}\left(\frac{\left|\mathcal{H}_{(l)}(1)\right|}{x}\right) \frac{\left|\mathcal{H}_{(l)}(1)\right|}{x^{2}},
\end{aligned}
$$

where the PDF $f_{l}(x)$ is given in (9).

Since the compensation in the SO-TCSM, $\left|\mathfrak{C}_{p}\left(\mathbf{v}_{a}\right)\right|$, is a random variable concerning all TAs are activated at the same probability, the PDF of $\left|\mathfrak{C}_{p}\left(\mathbf{v}_{a}\right)\right|$ is expressed as

$$
\begin{aligned}
\nu(x) & =\frac{1}{M} \sum_{l=1}^{M} \nu_{l}(x) \\
& =\frac{1}{M x^{2}} \sum_{l=1}^{M}\left|\mathcal{H}_{(l)}(1)\right| f_{l}\left(\frac{\left|\mathcal{H}_{(l)}(1)\right|}{x}\right) .
\end{aligned}
$$

Then, the PDF of the transmit power $P_{t}=\left|\mathfrak{C}_{p}\left(\mathbf{v}_{a}\right)\right|^{2}$ is calculated by

$$
\begin{aligned}
\phi(x) & =\nu\left(x^{\frac{1}{2}}\right)\left|\frac{d x^{\frac{1}{2}}}{d x}\right|=\frac{1}{2} \nu\left(x^{\frac{1}{2}}\right) x^{-\frac{1}{2}} \\
& =\frac{1}{2 M x^{\frac{3}{2}}} \sum_{l=1}^{M}\left|\mathcal{H}_{(l)}(1)\right| f_{l}\left(\frac{\left|\mathcal{H}_{(l)}(1)\right|}{x^{\frac{1}{2}}}\right) .
\end{aligned}
$$

The numerical results of $\phi(x)$ in (13) are compared with the simulation results over $10^{5}$ channel realisations in Fig. 9, for the PDF of the original transmit power $P_{t}$ without any constraint, in the SO-TCSM systems with QPSK and $M=2,4,8$ TAs. These comparisons verify the accuracy of our theoretical derivations for the PDFs in (9)-(13). 


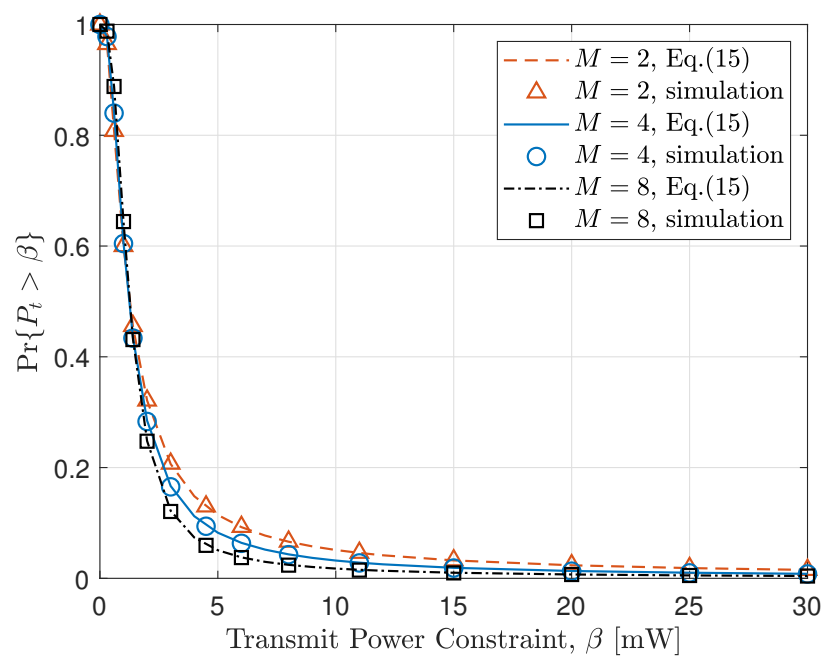

Fig. 10. The probability that transmit power is higher than the constraint $\beta$ for the SO-TCSM with QPSK and $M=2,4,8$ TAs.

\section{B. Transmit Power Clipped off by Constraint}

The transmit power clipped off by the maximum power capability $\beta$ is expressed as

$$
P_{\beta}= \begin{cases}\beta, & P_{t}>\beta, \\ P_{t}, & P_{t} \leqslant \beta,\end{cases}
$$

where $P_{\beta}$ denotes the transmit power under the constraint $\beta$. Thus, the compensation value is scaled to $\sqrt{\beta / P_{t}} \mathfrak{C}_{p}\left(\mathbf{v}_{a}\right)$ if the transmit power $P_{t}>\beta$, which will affect the SO-TCSM performance.

The probability that the transmit power determined by the compensation, $P_{t}$, is higher than the constraint $\beta$ is given by

$$
\operatorname{Pr}\left\{P_{t}>\beta\right\}=\int_{\beta}^{\infty} \phi(x) d x,
$$

where $\phi(x)$ is given in (13). Numerical results of $\operatorname{Pr}\left\{P_{t}>\beta\right\}$ in (15) are reported in Fig. 10 and compared with the simulation results over $10^{5}$ channel realisations, for the SO-TCSM systems with QPSK and $M=2,4,8$ TAs. As is shown in this figure, this probability decreases with the increase in the number of TAs, $M$, specifically when $\beta$ is larger than the transmit power of a QPSK symbol, namely $1 \mathrm{~mW}$. There are two reasons behind this phenomenon: Firstly, more TAs available at Alice result in lower probability that the active channel is in a deep fade; and secondly, more TAs lead to lower symbol energy of $\mathcal{O}(4 \hat{m}-3)$ in the target constellation. Moreover, this figure reveals that the probability $\operatorname{Pr}\left\{P_{t}>\beta\right\}$ plunges to the floor when $\beta$ increases to $5 \mathrm{~mW}$. Actually, the transmit power is lower than $5 \mathrm{~mW}$ in over $90 \%$ of the channel realisations, and lower than 2 $\mathrm{mW}$ in over $70 \%$ of the channel realisations, which means the required transmit power to meet the optimised compensation is acceptable. In addition, the probability $\operatorname{Pr}\left\{P_{t}>\beta\right\}$ approaches $10^{-3}$ at $\beta=30$. That is, it is almost impossible for the required transmit power to be higher than $30 \mathrm{~mW}$ and, thus, almost all required transmit power is attainable if the constraint $\beta$ is set to $30 \mathrm{~mW}$.

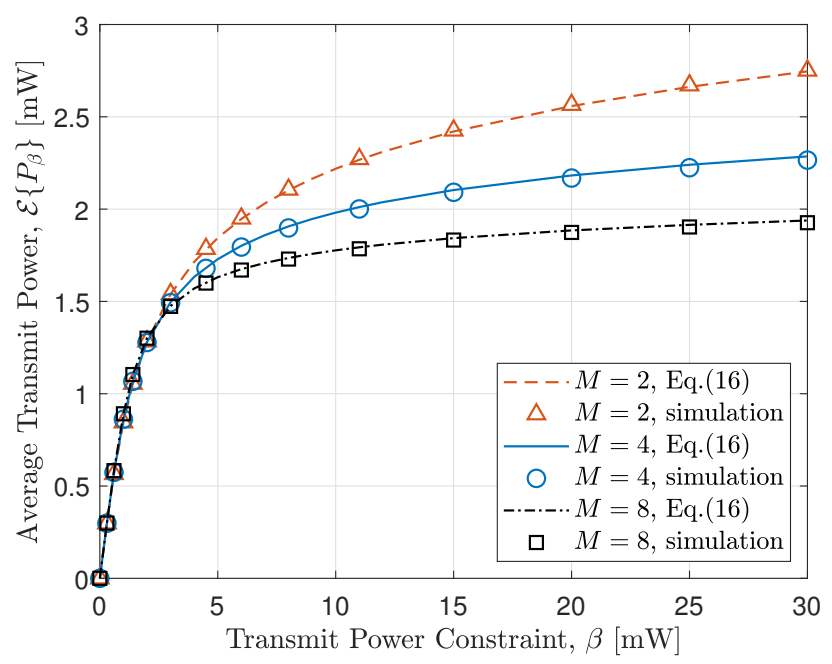

Fig. 11. Average transmit power $\mathcal{E}\left\{P_{\beta}\right\}$ of the SO-TCSM with QPSK and $M=2,4,8$ TAs, subject to the constraint $\beta$.

Without any constraint, i.e., $\beta=\infty$, the average transmit power of the SO-TCSM is obtained by $\mathcal{E}\left\{P_{t}\right\}=\mathcal{E}\{\mid \mathcal{O}(4 \hat{m}-$ $\left.3) /\left.\left(h_{\sharp \hat{m}} s_{\sharp 1}\right)\right|^{2}\right\}, \hat{m}=1,2, \cdots, M$. With the constraint $\beta$, the actual transmit-power $P_{\beta}$ is clipped to this limiting value when $P_{t}>\beta$. Based on (14), the average transmit power under the constraint $\beta$ is given by

$$
\begin{aligned}
\mathcal{E}\left\{P_{\beta}\right\} & =\int_{0}^{\beta} x \phi(x) d x+\beta \cdot \operatorname{Pr}\left\{P_{t}>\beta\right\} \\
& =\int_{0}^{\beta} x \phi(x) d x+\beta \int_{\beta}^{\infty} \phi(x) d x,
\end{aligned}
$$

where $\phi(x)$ is given in (13). Numerical results of $\mathcal{E}\left\{P_{\beta}\right\}$ in (16) are plotted in Fig. 11 and compared with the simulation results over $10^{5}$ channel realisations, where Alice adopts QPSK and $M=2,4,8$ TAs. As is shown in this figure, the average transmit power gets lower as the number of TAs, $M$, increases. This phenomenon is a counterpart of that in Fig. 10, and the reasons behind them are the same. Moreover, the average transmit power is convergent as the constraint $\beta$ increases. In the case of highest transmit power required, i.e., with $M=2$ TAs, the average transmit power converges to a point below $3 \mathrm{~mW}$, which manifests that a constraint of large $\beta$ can hardly affect the SO-TCSM transmissions, since the probability $\operatorname{Pr}\left\{P_{t}>\beta\right\}$ is extremely low in the case of large $\beta$.

To further exhibit the relation between the average transmit power $\mathcal{E}\left\{P_{\beta}\right\}$ and the constraint $\beta$, the peak-to-average power ratio (PAPR) of the SO-TCSM subject to the transmit power constraint is given by

$$
\begin{aligned}
\mathrm{PAPR} & =\frac{\beta}{\mathcal{E}\left\{P_{\beta}\right\}} \\
& =\frac{\beta}{\int_{0}^{\beta} x \phi(x) d x+\beta \int_{\beta}^{\infty} \phi(x) d x},
\end{aligned}
$$

where $\phi(x)$ is given in (13). Numerical results of the PAPR in (17) are plotted versus $\beta$ in Fig. 12 and compared with the simulation results over $10^{5}$ channel realisations, where Alice 


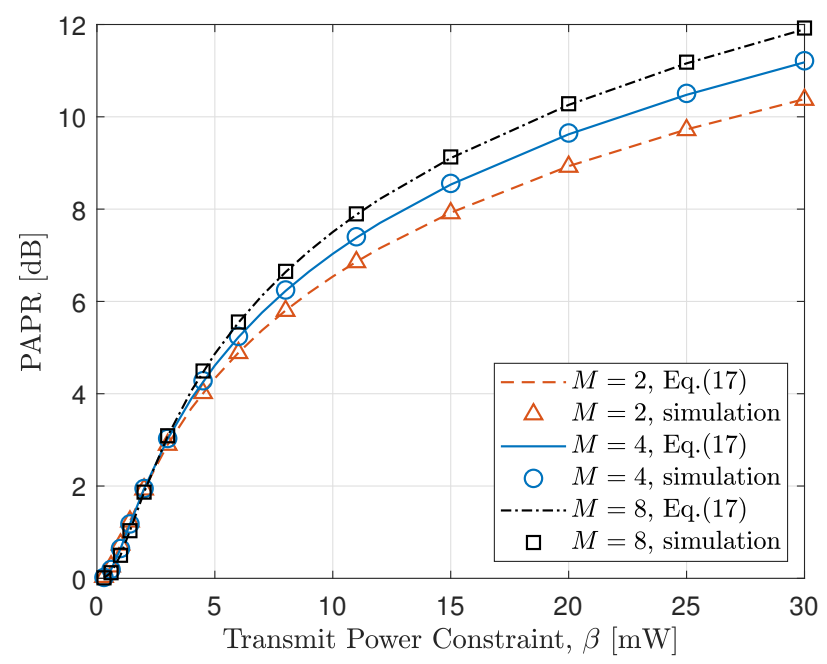

Fig. 12. PAPR of the SO-TCSM subject to the transmit power constraint $\beta$ for QPSK with $M=2,4,8$ TAs.

transmits QPSK symbols through activating one of $M=2,4,8$ TAs. The PAPR gets larger with the increase in the number of TAs, $M$, because the average transmit power gets lower as $M$ increases. Furthermore, the PAPR increases along with the increase in $\beta$, since the peak transmit power herein equals to $\beta$ and the average transmit power almost keeps constant in the case of large $\beta$.

From Figs. 10, 11, and 12, we may find that, when the transmit power constraint $\beta$ ranges from $5 \mathrm{~mW}$ to $20 \mathrm{~mW}$, the system design with SO-TCSM is feasible and economical concerning the transmit power consumption and the probability of sub-optimal solutions resulted from clipped transmit power. In this circumstance, the impact of the transmit power constraint on the BER performance of the SO-TCSM scheme is quantified in Fig. 13, where Alice adopts QPSK with $M=2,4,8$ TAs and the transmit power constraint is set to $\beta=5,10 \mathrm{~mW}$. The case of SO-TCSM without any transmit power constraint, i.e., $\beta=\infty$, is also shown for comparison. As anticipated, the BER performance of the SO-TCSM is not affected by the transmit power constraint at low SNRs. However, at high SNRs, the SO-TCSM performance gets better as the constraint value $\beta$ increases and the BER converges to the case of $\beta=\infty$. From Fig. 13, we may also find that the same constraint $\beta$ has less impact on the BER performance of the SO-TCSM with more TAs.

\section{Keeping Silent in Deep Fades}

In practice, to maintain the SO-TCSM performance at the level of no transmit power constraint, we may skip over the channel realisation in a deep fade, i.e., by keeping Alice silent when the required transmit power $P_{t}$ is higher than the constraint $\beta$. This skipping contributes to the improvement of energy efficiency as well, since no transmit power is wasted in deep fades. In this case, the average transmit power of the

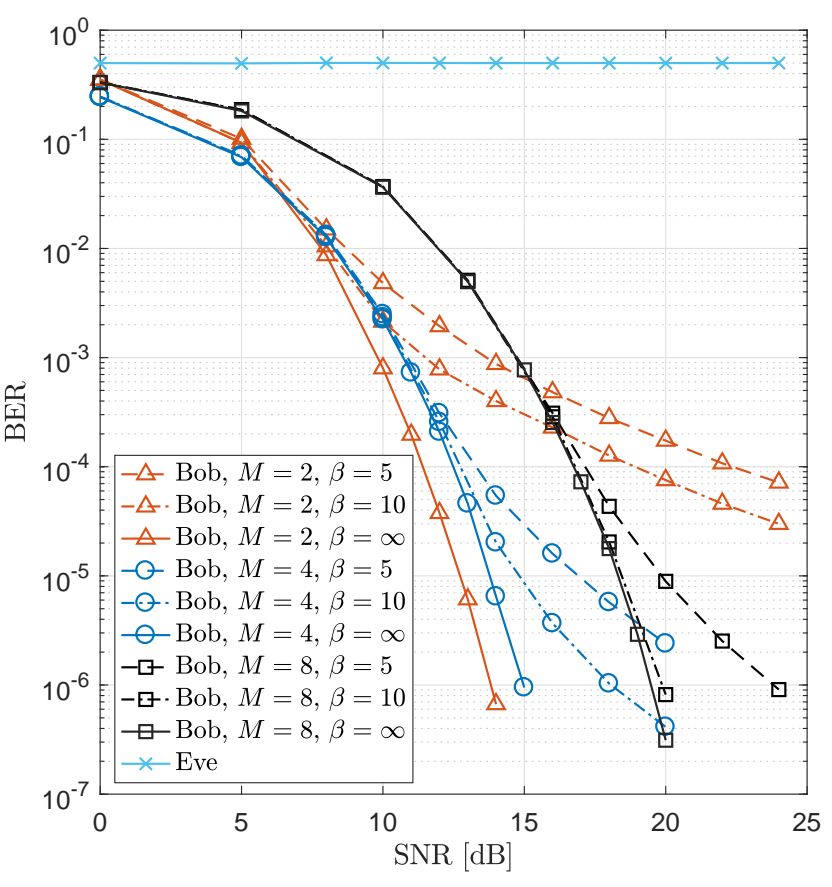

Fig. 13. BER of the SO-TCSM subject to the transmit power constraint $\beta$ for QPSK with $M=2,4,8$ TAs.

SO-TCSM is calculated by

$$
\mathcal{E}\left\{P_{t} \mid P_{t} \leqslant \beta\right\}=\frac{\int_{0}^{\beta} x \phi(x) d x}{\int_{0}^{\beta} \phi(x) d x},
$$

where $\phi(x)$ is given in (13). Numerical results of $\mathcal{E}\left\{P_{t} \mid P_{t} \leqslant \beta\right\}$ in (18) versus the transmit power constraint $\beta$ are compared with the simulation results over $10^{5}$ channel realisations in Fig. 14, where Alice adopts QPSK with $M=2,4,8$ TAs. In general, when the constraint $\beta$ is a small value, the average transmit power is almost the same for various $M$. However, in the region of large $\beta$, the average transmit power gets lower as the number of TAs, $M$, increases. Moreover, the average transmit power of the SO-TCSM skipping deep fades, $\mathcal{E}\left\{P_{t} \mid P_{t} \leqslant \beta\right\}$, is also convergent as $\beta$ increases and, compared with the average transmit power $\mathcal{E}\left\{P_{\beta}\right\}$ in Fig. 11, skipping over the channel realisation in deep fades does save considerable power consumption while achieving better BER performance.

However, the skipping over deep-fading channel realisations results in lower transmission rate. The transmission rate of the SO-TCSM skipping deep fades, denoted by $R_{\beta}$, is achieved at

$$
R_{\beta}=R \cdot \operatorname{Pr}\left\{P_{t} \leqslant \beta\right\}=R \int_{0}^{\beta} \phi(x) d x,
$$

where $R$ is the transmission rate of the SO-TCSM without skipping deep fades, and $\phi(x)$ is given in (13). In Fig. 15, the transmission rates of the SO-TCSM skipping deep fades given in (19) are plotted and compared with the simulation results over $10^{5}$ channel realisations, for the cases that Alice adopts $M=2,4,8$ TAs and QPSK with 2/3-, 2/4-, 3/5-rate trellis coding, where $R=2,2,3 \mathrm{bits} / \mathrm{sec} / \mathrm{Hz}$, respectively. As is shown in this figure, the transmission rate of the SO-TCSM skipping 


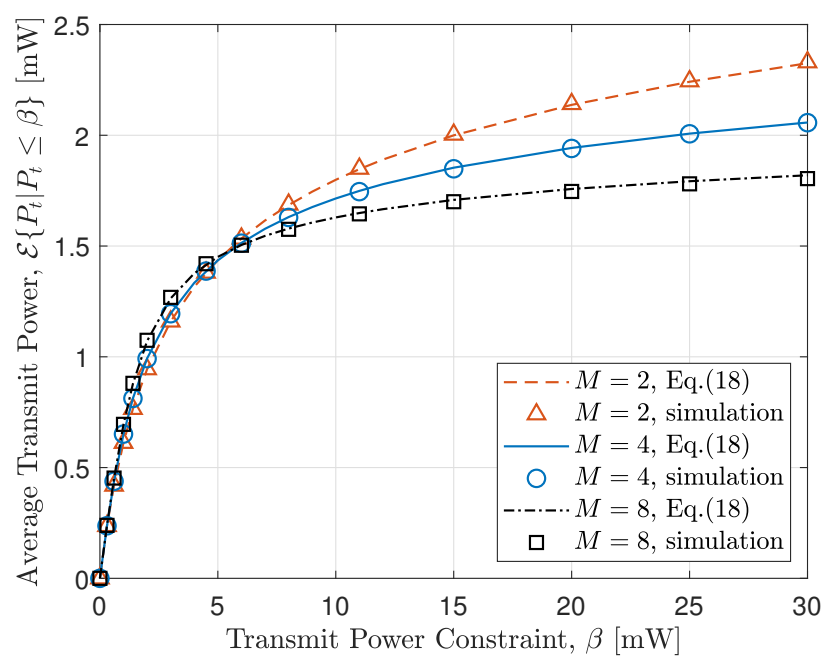

Fig. 14. Average transmit power $\mathcal{E}\left\{P_{t} \mid P_{t} \leqslant \beta\right\}$ of the SO-TCSM skipping deep fades, with QPSK and $M=2,4,8$ TAs.

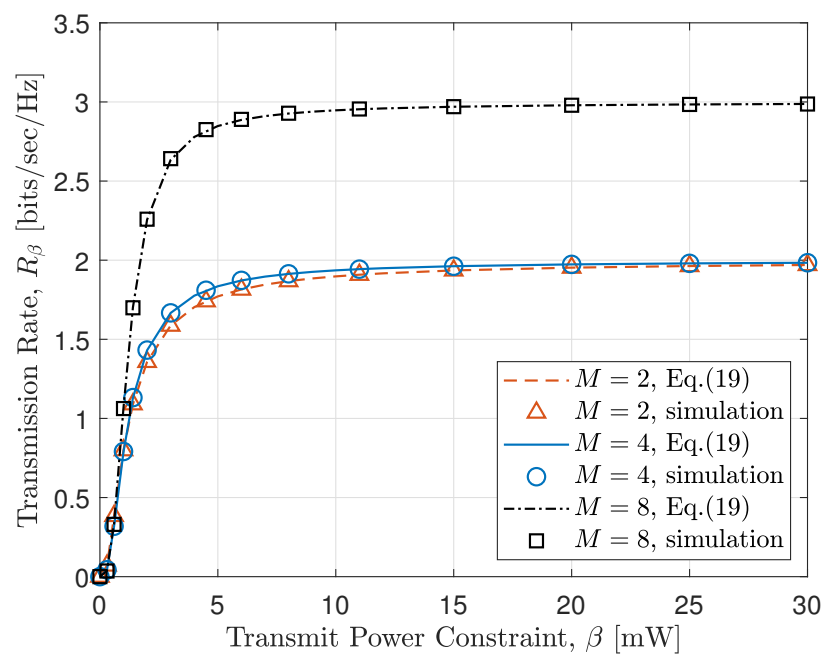

Fig. 15. Transmission rates of the SO-TCSM skipping deep fades, with QPSK and $M=2,4,8$ TAs relying on $2 / 3-, 2 / 4-, 3 / 5$-rate trellis coding.

over the channel realisation in deep fades converges to $R$ as the constraint $\beta$ increases, because $\lim _{\beta \rightarrow \infty} \operatorname{Pr}\left\{P_{t}>\beta\right\}=0$ and hence $\lim _{\beta \rightarrow \infty} \operatorname{Pr}\left\{P_{t} \leqslant \beta\right\}=1$. As such, the transmission rate of the SO-TCSM is determined by the transmit power constraint $\beta$ in this case.

\section{Practical ScEnarios}

In this section, the SO-TCSM is exploited in more practical scenarios, where the legitimate link is degraded by estimated CSI and Eve's channel is highly correlated with Bob's.

\section{A. Legitimate Channel Estimation}

In practice, perfect CSI is unavailable and the CSI is estimated through pilot observations. Herein, both Alice and Bob use the minimum mean square error (MMSE) estimation to

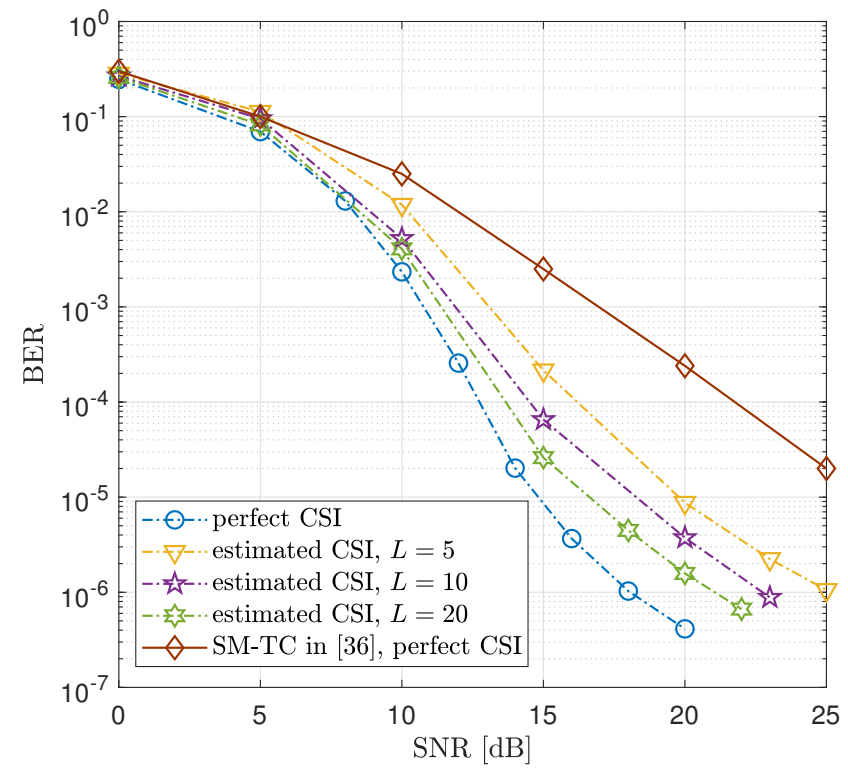

Fig. 16. The impact of estimated CSI on Bob's BER in the SO-TCSM system with QPSK and $M=4$ TAs.

obtain the CSI. In detail, the estimated CSI between the $m^{\text {th }}$ TA of Alice and Bob is calculated using [47], [48]

$$
\hat{h}_{m}=\sigma_{H}^{2} \mathbf{x}_{\mathrm{p}}^{\mathrm{T}}\left(\sigma_{H}^{2} \mathbf{x}_{\mathrm{p}} \mathbf{x}_{\mathrm{p}}^{\mathrm{T}}+\sigma_{W}^{2} \mathbf{I}_{L}\right)^{-1} \mathbf{y}_{\mathrm{p}, m},
$$

where the $L \times 1$ vector $\mathbf{x}_{\mathrm{p}}$ contains $L$ pilot symbols and $\mathbf{I}_{L}$ denotes the $L \times L$ identity matrix. The $L \times 1$ vector $\mathbf{y}_{p, m}$ contains the received signals, i.e., $\mathbf{y}_{\mathrm{p}, m}=h_{m} \mathbf{x}_{\mathrm{p}}+\mathbf{w}_{\mathrm{p}, m}$, where $h_{m}$ is the perfect CSI and $\mathbf{w}_{\mathrm{p}, m} \sim \mathcal{C N}\left(0, \sigma_{W}^{2}\right)$ is the received AWGN. The MMSE is $1 /\left(1 / \sigma_{H}^{2}+L / \sigma_{W}^{2}\right)$, which is reduced with the increase in the number of pilot symbols, $L$.

The impact of estimated CSI with $L=5,10,20$ on Bob's BER performance is investigated in Fig. 16, where Alice adopts QPSK and $M=4$ TAs with the transmit power clipped off by the constraint $\beta=10 \mathrm{~mW}$. As is shown in this figure, Bob's BER performance with estimated CSI gets better if more pilot symbols are used in the channel estimation. Moreover, although with estimated CSI and transmit power constraint, the SO-TCSM achieves better performance than the SM-TC in [36] with perfect CSI.

\section{B. Correlation between Eve and Bob}

Herein, we consider the scenario where Eve is located near Bob and, thus, her channel is spatially correlated with Bob's. The impact of this scenario on the security performance of our SO-TCSM is investigated.

With Kronecker product, the spatially correlated channels are modelled as [48]

$$
\left[\begin{array}{l}
\mathbf{h}_{\mathrm{sc}} \\
\mathbf{g}_{\mathrm{sc}}
\end{array}\right]=\mathbf{R}_{\mathrm{rx}}^{\frac{1}{2}}\left[\begin{array}{l}
\mathbf{h} \\
\mathbf{g}
\end{array}\right]
$$

where the $1 \times M$ vectors $\mathbf{h}_{\mathrm{sc}}$ and $\mathbf{g}_{\mathrm{sc}}$ are Bob's and Eve's channels of spatial correlation. The $1 \times M$ vectors $\mathbf{h}$ and $\mathbf{g}$ are independent channels of Bob and Eve. The $2 \times 2$ matrix $\mathbf{R}_{\mathrm{rx}}=$ 


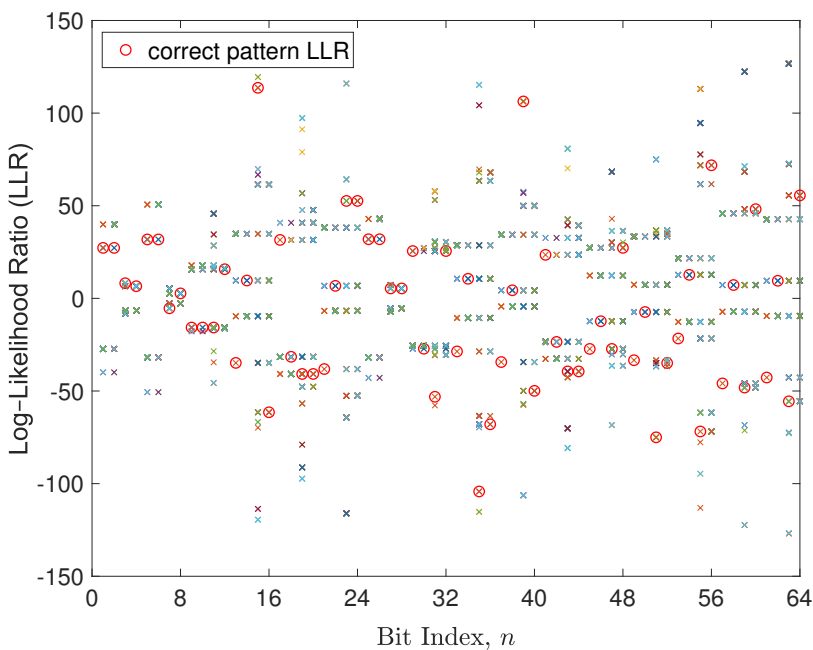

Fig. 17. Eve's LLRs using brute-force search in the SO-TCSM with QPSK and $M=4$ TAs, subject to a high correlation between Eve and Bob, $\lambda=0.9$.

$\left[\begin{array}{ll}1 & \lambda \\ \lambda & 1\end{array}\right]$ is the correlation matrix and $\lambda$ is the the correlation factor between Bob and Eve.

To take advantage of the spatial correlation with Bob, Eve is assumed to exploit a powerful detector with a brute-force search of all possible mapping and compensation patterns, for the recovery of Alice's information. Based on her wiretapped signals $y_{\mathrm{E}}$ given in (2), Eve calculates the log-likelihood ratio (LLR) for the $n^{\text {th }}$ bit in $\mathbf{v}=\left[\mathbf{v}_{a}, \mathbf{v}_{d}\right]$, denoted by $\mathbf{v}(n)$, using

$$
L L R_{\mathrm{E}}(n)=\ln \frac{\sum_{\mathbf{v}(n)=0} \exp \left(-\frac{\mid y_{\mathrm{E}}-g_{\left.\mathfrak{T}_{\mathrm{E}}\left(\mathbf{v}_{a}\right) \mathfrak{M}_{\mathrm{E}}\left(\mathbf{v}_{d}\right)\right|^{2}}}{\sigma_{W}^{2}}\right)}{\sum_{\mathbf{v}(n)=1} \exp \left(-\frac{\left|y_{\mathrm{E}}-g_{\mathfrak{T}_{\mathrm{E}}\left(\mathbf{v}_{a}\right)} \mathfrak{M}_{\mathrm{E}}\left(\mathbf{v}_{d}\right)\right|^{2}}{\sigma_{W}^{2}}\right)},
$$

by a brute-force search of all possible patterns for $\mathfrak{T}_{\mathrm{E}}(\cdot)$ and $\mathfrak{M}_{\mathrm{E}}(\cdot)$. In the SO-TCSM with QPSK and $M=4$ TAs, Eve's LLRs pertaining to all possible mapping patterns are shown in Fig. 17, for a high correlation between Eve and Bob with $\lambda=0.9$. As is shown in this figure, although the spatial correlation between Eve's and Bob's channels is very high, Eve cannot pick up the correct mapping and compensation pattern because she does not have any basis for the selection. The probability that Eve's maximum LLR matches the correct pattern is very low, equal to $\operatorname{Pr}\left\{\mathfrak{T}_{\mathrm{E}}(\cdot)=\mathfrak{T}_{p}(\cdot), \mathfrak{M}_{\mathrm{E}}(\cdot)=\right.$ $\left.\mathfrak{M}_{q}(\cdot)\right\}=(1 / P)(1 / Q)=1 / 96$.

As the brute-force search does not work for Eve, we assume Eve knows the three steps through which Alice formats the transmitted signals, i.e., from setting the TA index and QPSK mapping patterns to setting the compensation pattern. Then, based on her own channel coefficient that is highly correlated with Bob's, Eve attempts to anticipate the mapping and compensation patterns in the legitimate link, which is a better basis than random selection with the brute-force search. Using the anticipated mapping and compensation patterns for the recovery of Alice's information, Eve's BER performance is investigated in Fig. 18, for the SO-TCSM with QPSK and $M=2,4,8$

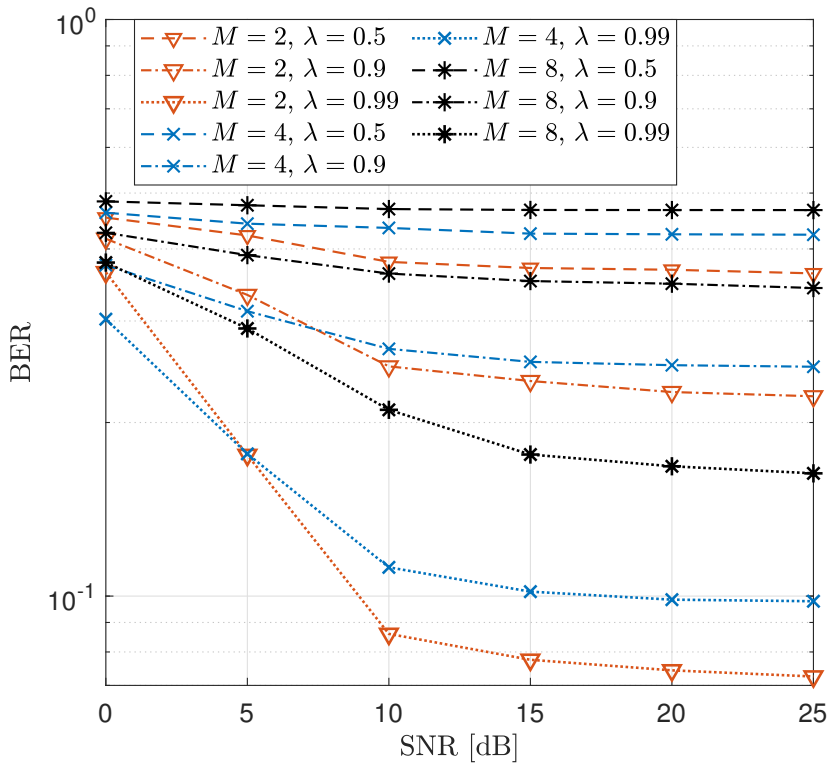

Fig. 18. Eve's BER using anticipated mapping and compensation patterns in the SO-TCSM with QPSK and $M=2,4,8$ TAs, subject to the spatial correlation between Eve and Bob, $\lambda=0.5,0.9,0.99$.

TAs, where the spatial correlation between Eve and Bob, $\lambda$ is set to $0.5,0.9$, and 0.99 . As is shown in this figure, although Eve's BER performance gets better with higher correlation, it is still much worse than Bob's and a floor effect arises as the SNR increases. The main reason behind this is that a slight difference between Bob's and Eve's channels result in different mapping and compensation patterns. With the increase in $M$, the chance that Eve's anticipated mapping and compensation patterns are different from those in the legitimate link gets higher, which explains why Eve's performance gets worse as $M$ increases.

\section{CONCLUSION AND DISCUSSION}

In this paper, a novel trellis coded SM design, referred to as SO-TCSM, was proposed for the purpose of PLS, where Alice varied the SM mapping pattern based on the legitimate CSI to optimise the Euclidean distances between the resultant SO-TCSM symbols. Since Eve had no knowledge on the legitimate CSI, she could not unveil the SM mapping pattern and, therefore, always failed to decode the confidential information. Moreover, Alice did not need to know Eve's CSI, which ensured the flexibility of the PLS implementation. Subsequently, the SOTCSM symbols were compensated to approach a predetermined target constellation of maximised Euclidean distances, which further improved Bob's decoding performance while enhancing the PLS. Compared with conventional trellis coded SM methods, the proposed SO-TCSM achieved better performance in the legitimate link with lower decoding complexity.

Concerning the energy utilisation efficiency, a constraint was set to control the transmit power for the SO-TCSM compensation in deep fades and the impact of this constraint on the SO-TCSM performance was investigated. Two transmission strategies were discussed for the required transmit power being higher than the constraint: $(i)$ clipping the transmit power to the 
constraint, and (ii) keeping silent to skip over the deep fades. To adopt which strategy might be determined by the tradeoff among the quality of service, battery life and latency requirements, since our investigations substantiated that the latter strategy achieved better BER performance with lower average transmit power and lower transmission rate than the former one.

Furthermore, the SO-TCSM was exploited in more practical scenarios with estimated CSI in the legitimate link and high correlation between Bob and Eve. The performance investigations in these scenarios illustrated the SO-TCSM with estimated CSI and transmit power constraint still outperforms the conventional SM-TC with perfect CSI, and the security is still guaranteed by the SO-TCSM even if Eve's channels are highly correlated with Bob's.

In addition, the trellis codes utilised in the studies we initiated here were not optimised for the SO-TCSM design but transferred from the classic ones for the traditional TCM design. Thus, the optimisation of the trellis encoding structures and the exploitation of turbo trellis coding are to be pursued for general SO-TCSM designs, aiming to achieve better performance in the legitimate link.

\section{REFERENCES}

[1] C. E. Shannon, "Communication theory of secrecy systems", Bell Labs Tech. J., vol. 28, no. 4, pp. 656-715, Oct. 1949.

[2] A. D. Wyner, "The wire-tap channel", Bell Syst. Tech. J., vol. 54, no. 8, pp. 1355-1387, 1975.

[3] A. Mukherjee, S. Fakoorian, J. Huang, and A. Swindlehurst, "Principles of physical layer security in multiuser wireless networks: A survey", IEEE Commun. Surveys Tuts., vol. 16, no. 3, pp. 1550-1573, 3rd Quart. 2014.

[4] J. Hamamreh, H. Furqan, and H. Arslan, "Classifications and applications of physical layer security techniques for confidentiality: A comprehensive survey", IEEE Commun. Surveys Tuts., vol. 21, no. 2, pp. 1773-1828, 2nd Quart. 2019.

[5] M. Bloch, J. Barros, M. Rodrigues, and S. McLaughlin, "Wireless information-theoretic security", IEEE Trans. Inf. Theory, vol. 54, no. 6, pp. 2515-2534, Jun. 2008.

[6] P. Gopala, L. Lai, and H. El Gamal, "On the secrecy capacity of fading channels", IEEE Trans. Inf. Theory, vol. 54, no. 10, pp. 4687-4698, Oct. 2008.

[7] A. O. Hero, "Secure space-time communication", IEEE Trans. Inf. Theory, vol. 49, no. 12, pp. 3235-3249, Dec. 2003.

[8] P. Lin, S. Lai, S. Lin, and H. Su, "On secrecy rate of the generalized artificial-noise assisted secure beamforming for wiretap channels", IEEE J. Sel. Areas Commun., vol. 31, no. 9, pp. 1728-1740, Sept. 2013.

[9] F. Oggier and B. Hassibi, "The secrecy capacity of the MIMO wiretap channel", IEEE Trans. Inf. Theory, vol. 57, no. 8, pp. 4961-4972, Aug. 2011.

[10] D. Ng, E. Lo, and R. Schober, "Robust beamforming for secure communication in systems with wireless information and power transfer", IEEE Trans. Wireless Commun., vol. 13, no. 8, pp. 4599-4615, Aug. 2014.

[11] W. Liao, T. Chang, W. Ma, and C. Chi, "QoS-based transmit beamforming in the presence of eavesdroppers: An optimized artificial-noise-aided approach", IEEE Trans. Signal Process., vol. 59, no. 3, pp. 1202-1216, Mar. 2011.

[12] N. Zhang, N. Cheng, N. Lu, X. Zhang, J. W. Mark, and X. Shen, "Partner selection and incentive mechanism for physical layer security", IEEE Trans. Wireless Commun., vol. 14, no. 8, pp. 4265-4276, Aug. 2015.

[13] J. Zhu, R. Schober, and V. Bhargava, "Linear precoding of data and artificial noise in secure massive MIMO systems", IEEE Trans. Wireless Commun., vol. 15, no. 3, pp. 2245-2261, Mar. 2016.

[14] N. Yang, L. Wang, G. Geraci, M. Elkashlan, J. Yuan, and M. D. Renzo, "Safeguarding 5G wireless communication networks using physical layer security", IEEE Commun. Mag., vol. 53, no. 4, pp. 20-27, Apr. 2015.

[15] F. Zhu, F. Gao, T. Zhang, K. Sun, and M. Yao, "Physical-layer security for full duplex communications with self-interference mitigation", IEEE Trans. Wireless Commun., vol. 15, no. 1, pp. 329-340, Jan. 2016.
[16] R. Zhang, X. Cheng, and L. Yang, "Cooperation via spectrum sharing for physical layer security in device-to-device communications underlaying cellular networks", IEEE Trans. Wireless Commun., vol. 15, no. 8, pp. 56515663, Aug. 2016.

[17] Y. Liu, Z. Qin, M. Elkashlan, Y. Gao, and L. Hanzo, "Enhancing the physical layer security of non-orthogonal multiple access in large-scale networks", IEEE Trans. Wireless Commun., vol. 16, no. 3, pp. 1656-1672, Mar. 2017.

[18] F. Zhou, Z. Li, J. Cheng, Q. Li, and J. Si, "Robust AN-aided beamforming and power splitting design for secure MISO cognitive radio with SWIPT", IEEE Trans. Wireless Commun., vol. 16, no. 4, pp. 2450-2464, Apr. 2017.

[19] X. Chen, D. Ng, W. Gerstacker, and H. Chen, "A survey on multipleantenna techniques for physical layer security", IEEE Commun. Surveys Tuts., vol. 19, no. 2, pp. 1027-1053, 2nd Quart. 2017.

[20] Y. Yang and B. Jiao, "Information-guided channel-hopping for high data rate wireless communication", IEEE Commun. Lett., vol. 12, no. 4, pp. 225-227, Apr. 2008.

[21] M. Di Renzo, H. Haas, and P. M. Grant, "Spatial modulation for multipleantenna wireless systems: A survey", IEEE Commun. Mag., vol. 49, no. 12, pp. 182-191, Dec. 2011.

[22] Y. Yang and S. Aissa, "Information guided channel hopping with an arbitrary number of transmit antennas", IEEE Commun. Lett., vol. 16, no. 10, pp. 1552-1555, Oct. 2012.

[23] C. Wu, Y. Xiao and P. Yang, "Covert information embedded spatial modulation", IEEE Commun. Lett., doi: 10.1109/LCOMM.2020.3008430.

[24] L. Wang, S. Bashar, Y. Wei, and R. Li, "Secrecy enhancement analysis against unknown eavesdropping in spatial modulation", IEEE Commun. Lett., vol. 19, no. 8, pp. 1351-1354, Aug. 2015.

[25] C. Liu, L. Yang, and W. Wang, "Secure spatial modulation with a fullduplex receiver", IEEE Wireless Commun. Lett., vol. 6, no. 6, pp. 838-841, Dec. 2017.

[26] Y. Chen, L. Wang, Z. Zhao, M. Ma and B. Jiao, "Secure multiuser MIMO downlink transmission via precoding-aided spatial modulation", IEEE Commun. Lett., vol. 20, no. 6, pp. 1116-1119, Jun. 2016.

[27] Y. Huang, M. Wen, B. Zheng, X. Cheng, L. Yang, and F. Ji, "Secure precoding aided spatial modulation via transmit antenna selection", IEEE Trans. Veh. Technol., vol. 68, no. 9, pp. 8893-8905, Sept. 2019.

[28] F. Shu, Z. Wang, R. Chen, Y. Wu, and J. Wang, "Two high-performance schemes of transmit antenna selection for secure spatial modulation", IEEE Trans. Veh. Technol., vol. 67, no. 9, pp. 8969-8973, Sept. 2018.

[29] G. Xia, Y. Lin, T. Liu, F. Shu, and L. Hanzo, "Transmit antenna selection and beamformer design for secure spatial modulation with rough CSI of Eve", IEEE Trans. Wireless Commun., Early Access, doi: 10.1109/TWC.2020.2985968.

[30] Y. Yang and M. Guizani, "Mapping-varied spatial modulation for physical layer security: Transmission strategy and secrecy rate", IEEE J. Sel. Areas Commun., vol. 36, no. 4, pp. 877-889, Apr. 2018.

[31] G. Ungerboeck, "Channel coding with multilevel/phase signals", IEEE Trans. Inf. Theory, vol. 28, no. 1, pp. 55-67, 1982.

[32] R. Mesleh, M. D. Renzo, H. Haas, and P. M. Grant, "Trellis coded spatial modulation", IEEE Trans. Wireless Commun., vol. 9, no. 7, pp. 2349-2361, Jul. 2010.

[33] C. Vladeanu, "Turbo trellis-coded spatial modulation", in Proc. IEEE Global Commun. Conf. (GLOBECOM), pp. 4024-4029, 2012.

[34] Y. Zhou, D. Yuan, X. Zhou, and H. Zhang, "Trellis Coded Generalized Spatial Modulation", in Proc. IEEE Veh. Technol. Conf. (VTC Spring), Seoul, pp. 1-5, 2014

[35] S. Long, P. Tsai, Y. Huang, and I. Lai, "Trellis coded generalized spatial modulation with spatial multiplexing", in Proc. Asia-Pacific Signal and Inf. Process. Asso. Annual Summit and Conf. (APSIPA), Dec. 2017.

[36] E. Basar, U. Aygolu, E. Panayirci, and H. V. Poor, "New Trellis Code Design for Spatial Modulation", IEEE Trans. Wireless Commun., vol. 10, no. 8, pp. 2670-2680, Aug. 2011.

[37] C. Vladeanu, "Spatial modulation with joint antenna index and symbol index turbo trellis coding", in Proc. Int. Symp. Signals, Circuits and Syst. (ISSCS), Oct. 2013.

[38] T. Hara, K. Ishibashi, S. Ng, and L. Hanzo, "Low-complexity generator polynomial search for turbo trellis-coded spatial modulation using symbolbased EXIT charts", in Proc. IEEE Int. Symp. Turbo Codes (ISTC), Dec. 2018.

[39] P. Yang, Y. Xiao, M. Xiao, Y. L. Guan, S. Li and W. Xiang, "Adaptive spatial modulation MIMO based on machine learning", IEEE J. Sel. Areas Commun., vol. 37, no. 9, pp. 2117-2131, Sept. 2019.

[40] S. Lin and D. Costello, Error Control Coding Fundamentals and Applications, $2^{\text {nd }}$ edition. Prentice-Hall Inc., 2004. 
[41] G. Foschini, R. Gitlin and S. Weinstein, "Optimization of TwoDimensional Signal Constellations in the Presence of Gaussian Noise", IEEE Trans. Commun., vol. 22, no. 1, pp. 28-38, Jan. 1974.

[42] A. Morello and V. Mignone, "DVB-S2: The second generation standard for satellite broad-band services", Proc. IEEE, vol. 94, no. 1, pp. 210-227, Jan. 2006.

[43] A. Hoglund, D. Van, T. Tirronen, O. Liberg, Y. Sui and E. Yavuz, "3GPP Release 15 Early Data Transmission”, IEEE Commun. Standards Mag., vol. 2, no. 2, pp. 90-96, Jun. 2018.

[44] D. MacKay, Information Theory, Inference, and Learning Algorithms, Cambridge University Press, 2003.

[45] V. Kotagi, R. Thakur, S. Mishra and C. Murthy, "Breathe to save energy: Assigning downlink transmit power and resource blocks to LTE enabled IoT networks", IEEE Commun. Lett., vol. 20, no. 8, pp. 1607-1610, Aug. 2016.

[46] H. A. David and H. N. Nagaraja, "Order statistics", Encyclopedia Statistical Sci., vol. 67, no. 339, pp. 10897-10901, 2004.

[47] Y. Yang, N. Bonello, and S. Aissa, "An information-guided channelhopping scheme for block-fading channels with estimation errors", in Proc. IEEE Global Commun. Conf. (GLOBECOM), Miami, pp. 1-5, 2010.

[48] Y. Yang and S. Aissa, "Information-guided communications in MIMO systems with channel state impairments", Wirel. Commun. Mob. Comput., vol. 15, pp. 868-878, 2015.

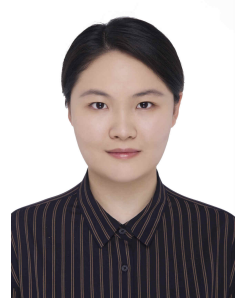

Mingxi Yin received the B.Eng. degree in electronics engineering from Beijing University of Posts and Telecommunications (BUPT), Beijing, China, in 2016. She is currently pursuing the Ph.D. degree with the Department of Electronics, Peking University, Beijing, China. Her research interests include wireless communications in the physical layer and wireless digital health.

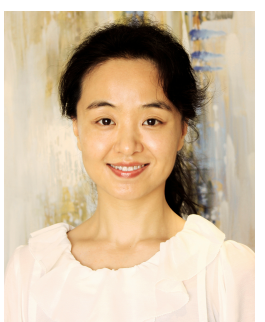

Yuli Yang (S'04-M'08-SM'19) received her Ph.D. degree in Communications \& Information Systems from Peking University in July 2007. Since Dec 2019, she has been with the University of Lincoln as a Senior Lecturer in Electrical/Electronic Engineering. From Jan 2010 to Dec 2019, she was with King Abdullah University of Science \& Technology, Melikşah University, and the University of Chester on various academic positions. Her industry experience includes working as a Research Scientist with Bell Labs Shanghai, from Aug 2007 to Dec 2009, and an Intern Researcher with Huawei Technologies, from June 2006 to July 2007. Her research interests include modelling, design, analysis and optimization of wireless systems and networks.

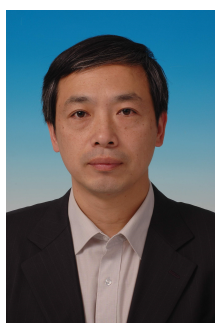

Bingli Jiao (M'05-SM'11) received the B.S. and M.S. degrees from Peking University, China, in 1983 and 1988, respectively, and the Ph.D. degree from Saarland University, Germany, in 1995 . He became an Associate Professor in 1995 and a Professor with Peking University in 2000. He currently is the director of Wireless Communication and Signal Processing Research Center, Peking University, Beijing, China. He is also a director of the Joint Laboratory for Advanced Communication Research between Peking University and Princeton University. His current research interests include full-duplex communications, information theory, and signal processing. $\mathrm{He}$ is a pioneer of co-frequency and co-time full-duplex as found in his early patent in 2006. 